Published in final edited form as:

Nat Rev Dis Primers. ; 5(1): 38. doi:10.1038/s41572-019-0087-y.

\title{
Paediatric and adult-onset male hypogonadism
}

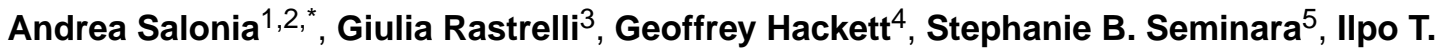 \\ Huhtaniemi ${ }^{6,7}$, Rodolfo A. Rey ${ }^{8}$, Wayne J. G. Hellstrom ${ }^{9}$, Mark R. Palmert ${ }^{10,11}$, Giovanni \\ Corona $^{3,12}$, Gert R. Dohle ${ }^{13}$, Mohit Khera ${ }^{14}$, Yee-Ming Chan ${ }^{15,16}$, Mario Maggi $i^{3,17}$
}

${ }^{1}$ Division of Experimental Oncology, Unit of Urology, URI, IRCCS Ospedale San Raffaele, Milan, Italy. ${ }^{2}$ Università Vita-Salute San Raffaele, Milan, Italy. ${ }^{3}$ Sexual Medicine and Andrology Unit Department of Experimental Clinical and Biomedical Sciences 'Mario Serio', University of Florence, Florence, Italy. ${ }^{4}$ Department of Urology, University of Bedfordshire, Bedfordshire, UK. ${ }^{5}$ Harvard Reproductive Sciences Center and Reproductive Endocrine Unit, Department of Medicine, Massachusetts General Hospital, Boston, MA, USA. ${ }^{6}$ Department of Surgery and Cancer, Imperial College London, Hammersmith Campus, London, UK. ${ }^{7}$ Department of Physiology, Institute of Biomedicine, University of Turku, Turku, Finland. ${ }^{8} \mathrm{Centro}$ de Investigaciones Endocrinológicas 'Dr César Bergadá' (CEDIE), CONICET - FEI — División de Endocrinología, Hospital de Niños R. Gutiérrez, Buenos Aires, Argentina. ${ }^{9}$ Department of Urology, Tulane University School of Medicine, New Orleans, LA, USA. ${ }^{10}$ Division of Endocrinology, The Hospital for Sick Children, Toronto, Ontario, Canada. ${ }^{11}$ Departments of Paediatrics and Physiology, University of Toronto, Toronto, Ontario, Canada. ${ }^{12}$ Endocrinology Unit, Medical Department, Azienda UsI Bologna Maggiore-Bellaria Hospital, Bologna, Italy. ${ }^{13}$ Department of Urology, Erasmus University Medical Centre, Rotterdam, Netherlands. ${ }^{14} \mathrm{Scott}$ Department of Urology, Baylor College of Medicine, Houston, TX, USA. ${ }^{15}$ Division of Endocrinology, Department of Pediatrics, Boston Children's Hospital, Boston, MA, USA. ${ }^{16}$ Department of Pediatrics, Harvard Medical School, Boston, MA, USA. ${ }^{17}$ Istituto Nazionale Biostrutture e Biosistemi (INBB), Rome, Italy.

\section{Abstract}

The hypothalamic-pituitary-gonadal axis is of relevance in many processes related to the development, maturation and ageing of the male. Through this axis, a cascade of coordinated activities is carried out leading to sustained testicular endocrine function, with gonadal testosterone production, as well as exocrine function, with spermatogenesis. Conditions impairing the hypothalamic-pituitary-gonadal axis during paediatric or pubertal life may result in delayed puberty. Late-onset hypogonadism is a clinical condition in the ageing male combining low

\footnotetext{
* salonia.andrea@hsr.it.

Author contributions

Introduction (A.S.); Epidemiology (G.R. and G.H.); Mechanisms/pathophysiology (S.B.S. and I.T.H.); Diagnosis, screening and prevention (R.A.R. and W.J.G.H.); Management (M.R.P., G.C. and G.R.D.); Quality of life (M.K.); Outlook (Y.-M.C. and M.M.); Overview of Primer (A.S.).

Competing interests

The authors declare no competing interests.

Publisher's note

Springer Nature remains neutral with regard to jurisdictional claims in published maps and institutional affiliations.
} 
concentrations of circulating testosterone and specific symptoms associated with impaired hormone production. Testosterone therapy for congenital forms of hypogonadism must be lifelong, whereas testosterone treatment of late-onset hypogonadism remains a matter of debate because of unclear indications for replacement, uncertain efficacy and potential risks. This Primer focuses on a reappraisal of the physiological role of testosterone, with emphasis on the critical interpretation of the hypogonadal conditions throughout the lifespan of the male individual, with the exception of hypogonadal states resulting from congenital disorders of sex development.

The hypothalamic-pituitary-gonadal (HPG) axis (FIG. 1) is of paramount importance in many processes related to the development, maturation and ageing of the male ${ }^{1}$. The pulsatile secretion of gonadotropin-releasing hormone $(\mathrm{GnRH})$ by the hypothalamus stimulates the biosynthesis of gonadotropins (glycoprotein polypeptide hormones secreted by the anterior pituitary gland) - namely, luteinizing hormone (LH) and follicle-stimulating hormone (FSH). LH (with gonadal testosterone and insulin-like factor 3 (INSL3)) sustains testicular endocrine function led by Leydig cells which is required for male genital development and differentiation throughout human sexual determination ${ }^{2}$. FSH, in turn, sustains testicular exocrine function led by Sertoli cells through spermatogenesis. The two Sertoli cell hormones, anti-Müllerian hormone (AMH) and inhibin B, participate in the regulation of genital masculinization and negative feedback regulation of FSH secretion, respectively. Likewise, the HPG axis has a key role in completing phenotypic differentiation and development of the fetus and male sexual maturation at puberty and into adulthood ${ }^{3}$.

Overall, congenital or acquired disturbances at any level of the HPG axis can lead to an impairment of reproductive function and the clinical syndrome of hypogonadism. Male hypogonadism is a disorder associated with decreased functional activity of the testes, with decreased production of androgens (steroid hormones that regulate male characteristics), inhibin B, AMH and/or impaired sperm production ${ }^{4}$. Hypogonadism can be caused by a primary testicular pathology (primary hypogonadism, otherwise known as hypergonadotropic hypogonadism) resulting from malfunction at the level of the testes due to a genetic cause, injury, inflammation or infection (BOXES 1,2). Conversely, hypothalamic and/or pituitary failures lead to secondary hypogonadism (also called central hypogonadism or hypogonadotropic hypogonadism), which is most often caused by genetic defects, neoplasm or infiltrative disorders (BOXES 1,2).

Signs and symptoms of hypogonadism depend on the age of onset, the severity of androgen deficiency and the underlying cause of androgen deficiency ${ }^{3}$. In healthy childhood, testosterone is low and spermatozoa are not produced; thus, decreased Sertoli cell function, as reflected by low inhibin B and AMH, is the cardinal sign of hypogonadism before puberty. Conditions impairing the HPG axis that occur during paediatric or pubertal life may result in delayed puberty (defined as the lack of sexual maturation by an age at which 95$98 \%$ of the children have initiated sexual maturation $)^{5,6}$. In adulthood, this can manifest as testosterone deficiency, which is a clinical syndrome resulting from reduced testicular testosterone production or from reduced serum testosterone activity, in conjunction with clinical signs and symptoms. Spermatogenic failure is usually included as a sign of hypogonadism. When specifically associated with ageing, obesity or poor health, adult-onset 
hypogonadism is usually called late-onset hypogonadism ( $\mathrm{LOH})$. Depending on the causes, LOH can be persistent or potentially reversible (BOX 1).

This Primer focuses on a reappraisal of the physiological role of testosterone, with emphasis given to the critical interpretation of the hypogonadal conditions throughout the entire lifespan of the male, while considering as distinct the two periods of paediatric/pubertal and adult life in terms of both pathophysiology and clinical manifestations. Hypogonadal states resulting in congenital disorders of sex development fall outside the scope of this Primer.

\section{Epidemiology}

\section{Pubertal hypogonadism}

According to the age of onset, paediatric hypogonadism can manifest differently (BOX 3).

Clinical presentation of hypogonadism at birth is rare, with abnormal genitalia occurring in $\sim 1$ in 4,500 live births ${ }^{7}$. Hypogonadism established after birth, throughout childhood, is usually inapparent until pubertal age; at this point, the hypogonadal condition manifests as delayed puberty. The prevalence of delayed puberty in the general population has not been thoroughly assessed. In this context, it is important to note that the current definition of delayed puberty is statistical. In most textbooks and review articles, a cut-off of 14 years is cited; using this definition, early studies have found a prevalence of delayed puberty of $<2 \%$ among boys in the United States ${ }^{8}$. However, the real prevalence is likely higher because transient forms (such as constitutional delay of growth and puberty (CDGP) or reversible forms of congenital isolated secondary hypogonadism) may remain undiagnosed. Delayed puberty is caused by CDGP in $60 \%$ of cases, secondary hypogonadism of organic origin in $10 \%$ of cases, primary hypogonadism of organic origin in $\sim 7 \%$ of cases or functional hypogonadism (both primary and secondary) in $20 \%$ of cases ${ }^{9-12}$. Some examples of paediatric and pubertal hypogonadisms of functional origin, which include chronic diseases and excessive drug use causing physiological suppression of the HPG axis, are listed in BOX 2 (REFS $^{9-12}$ ). Type 1 diabetes mellitus is not usually associated with delayed puberty unless it is poorly controlled. However, in many of the chronic illnesses that can lead to a transient disorder, functional hypogonadism is rare, having an incidence between 1 per 10,000 and 1 per 100,000 per year over childhood and young age ${ }^{13-16}$.

Secondary hypogonadism.-Secondary hypogonadism can be congenital or acquired (BOX 2). Congenital forms can present as isolated secondary hypogonadism, in which only GnRH and/or gonadotropins levels are affected, whereas the rest of the hypothalamicpituitary hormone axis remains intact. Congenital secondary hypogonadism is rare, having a global prevalence of 1 case per 4,000-10,000 boys ${ }^{17}$. Kallmann syndrome represents $60 \%$ of these forms and has been recognized to be familial (X-linked, autosomal dominant or autosomal recessive) or sporadic ${ }^{18}$. In addition to secondary hypogonadism, patients with Kallmann syndrome also present with hypo-anosmia (lack of smell) and malformations (such as midline defects, unilateral renal agenesis, bimanual synkinesia or mirror movements, syndactyly and dental agenesis) ${ }^{17}$.

Combined congenital secondary hypogonadism, is characterized by the deficiency of multiple pituitary hormones and results from a wide variety of even more rare genetic 
conditions than isolated congenital hypogonadism, such as mutations of HESX1, PROP1, $L H X 3$ and $L H X 4$, which encode proteins involved in the HPG axis.

Acquired secondary hypogonadism could result from intracranial masses (such as craniopharyngioma) or infiltrative diseases (such as Langerhans cell histiocytosis) (BOX 2), both rare conditions, with a reported incidence of $<5.0$ per 1,000,000 per year, but characterized by a twofold higher prevalence during childhood than adulthood ${ }^{19,20}$. Other causes of acquired secondary hypogonadism are more typical in adult age. However, they can occur also in children and adolescents, delaying normal pubertal onset or progression. Of relevance, of boys with delayed puberty, the majority of cases are due to chronic diseases, such as hypothyroidism, growth hormone deficiency, Crohn's disease and severe asthma ${ }^{9}$. Malnutrition is described in 3\% of cases, whereas excessive exercise and anorexia nervosa as well as hyperprolactinaemia are less frequent in $\operatorname{males}^{9}$ (BOX 2).

Primary hypogonadism.-Klinefelter syndrome, a trisomy associated with a 47, XXY karyotype $^{21,22}$, is the most important genetic cause of primary hypogonadism, with a global prevalence of 1 per 500-1,000 live male births ${ }^{23}$. A wide range of clinical manifestations characterize Klinefelter syndrome, but low testis volume and less often slow pubertal development are commonly reported, and primary hypogonadism develops almost universally by late adolescence (resulting in a delayed accomplishment of pubertal development) or early adulthood ${ }^{24}$. Of note, Klinefelter syndrome is frequently undiagnosed; indeed, as few as $25 \%$ of patients with Klinefelter syndrome are accurately diagnosed and most of these diagnoses are not made until adulthood ${ }^{25}$. Nevertheless, this may be changing with increased use of prenatal testing and increased use of karyotyping of comparative genomic hybridization array in evaluating boys with learning and/or behavioural issues.

Acquired causes of primary hypogonadism during childhood or puberty include chemotherapy and radiotherapy. For instance, alkylating agents are associated with a spermatogenesis failure in $80-90 \%$ of cases, which is irreversible in the majority of cases ${ }^{26}$. In $\sim 10 \%$ of childhood cancer survivors, normal testosterone levels can be found with a normal development of secondary sexual characteristics ${ }^{26}$. After puberty, irradiation and chemotherapy may have even more severe adverse effects on testicular function.

\section{Adult-onset hypogonadism}

In healthy, young eugonadal men (defined as $<30$ years of age, with a normal testosterone production), serum testosterone levels range from 10.4 to $36.4 \mathrm{nmol} / 1$ (300-1,050 ng/dl), with a slight gradual decline after 40 years of age ${ }^{27}$ (FIG 1). Using a serum total testosterone level $<11 \mathrm{nmol} / 1$ (317 ng/dl) to define biochemical hypogonadism, the Baltimore Longitudinal Study of Aging (BLSA) reported that $12 \%, 20 \%, 30 \%$ and $50 \%$ of men in their 50s, 60s, 70s and 80s, respectively, are biochemically hypogonadal ${ }^{27}$. However, estimates regarding the prevalence of symptomatic hypogonadism vary widely. The European Male Ageing Study (EMAS) evaluated >3,000 men aged 40-79 years according to the combination of biochemistry (total testosterone level of $<11 \mathrm{nmol} / 1(317 \mathrm{ng} / \mathrm{dl})$ and a free testosterone level of $<0.22 \mathrm{nmol} / 1(6.3 \mathrm{ng} / \mathrm{dl})$ ) and specific symptoms (presence of 
erectile dysfunction, loss of morning erections and reduced sexual desire $)^{28}$. Results showed an overall prevalence of $2.1 \%$ in men aged 40-79 years and rates of $0.1 \%$ in $40-49$ year olds, $0.6 \%$ in $50-59$ year olds, $3.2 \%$ in $60-69$ year olds and $5.1 \%$ in $70-79$ year olds. Of note, $63 \%$ of men maintained physiological total testosterone $(>11 \mathrm{nmol} / 1)$ levels into old age (70-79 years of age), suggesting that hypogonadism is not a uniform sign of ageing. Using only biochemical criteria, central hypogonadism was found in $11.8 \%$ of the EMAS cohort, with $2 \%$ having primary hypogonadism and $9.5 \%$ having 'compensated hypogonadism' (defined as high LH, with normal total testosterone), a condition not associated with clear symptoms of hypogonadism but that may be a potential harbinger of primary hypogonadism ${ }^{29,30}$. Hence, $\mathrm{LOH}$ is a relatively common condition in the ageing male ( $>40$ years of age) - with a prevalence of $2-15 \%$ within the general population - and it is frequently associated with age-related comorbidities, such as obesity, metabolic syndrome and type 2 diabetes mellitus (T2DM) ${ }^{31,32}$.

\section{Mechanisms/pathophysiology}

\section{The physiology of the HPG axis}

An understanding of the role of the HPG axis throughout development, puberty and ageing and its role in male sex development is crucial in order to comprehend the pathophysiology of hypogonadism (FIG 2).

Infancy and childhood.-The initial differentiation of the testes in the embryo precedes the functional development of pituitary gonadotropins (BOX 4). At birth, gonadotropins and testicular hormone levels are low and increase during the first weeks and months of life ${ }^{33,34}$. Peak levels of LH, testosterone and INSL3 are reached during the third month ${ }^{4}$. Thereafter, serum gonadotropins and testosterone decline and remain low until the onset of puberty (FIG 2). During this relative physiological quiescence in childhood, basal gonadotropins and testosterone measurements do not reflect HPG axis function. Conversely, AMH and inhibin B levels increase progressively through infancy and remain high during childhood; their basal levels are most informative of Sertoli cell function ${ }^{4}$. Interestingly, the high androgen levels observed during fetal life and the postnatal period are not capable of inducing Sertoli cell maturation, full spermatogenesis and secondary sex characteristics as they do at puberty. The lack of androgen effect on Sertoli cells and spermatogenesis may be explained by the absence of androgen receptor expression in Sertoli cells over the first year of life. Indeed, the abnormal persistence of elevated testosterone beyond the first year results in signs of seminiferous tubule maturation (that is, a reduction in $\mathrm{AMH}$ and increase in inhibin $\mathrm{B})^{35}$.

Puberty.-Puberty in boys begins with a progressive increase in the pulse amplitude and frequency of gonadotropin release ${ }^{36}$. FSH induces the proliferation of immature Sertoli cells and boosts testicular volume from $2 \mathrm{ml}$ to $4 \mathrm{ml}$, the clinical landmark of pubertal onset that occurs at a mean age of 11.5 years ${ }^{37}$. Pubertal onset is considered delayed if it occurs between 14 years and 18 years of age, with the exact cut-off age depending on the considered guidelines ${ }^{38}$. LH induces a progressive increase in testicular androgen production; androgens together with FSH trigger Sertoli cell maturation. Consequently, Sertoli cell proliferation stops, AMH production declines and inhibin B secretion rises, as 
seen in the initial stages of puberty (that is, stages 2 and 3 following the classic description by Marshall and Tanner ${ }^{39,40}$ ). The overt increase in serum testosterone levels is a later event, particularly marked during Tanner stages 3 to $5\left(\mathrm{REF}^{41}\right)$. Germ cells undergo the complete spermatogenic process, leading to sperm production and to the overt increase in testis volume to $15-25 \mathrm{ml}$ (FIG. 2) in Tanner stages 4 and 5. FSH and spermatogenesis are essential for inhibin B production, which in turn acts as a negative feedback regulator of pituitary FSH secretion ${ }^{42}$. Thus, in puberty and adulthood, inhibin B is an extremely informative biomarker of testicular function because it reflects the whole pubertal maturation process (for example, FSH and testosterone action on Sertoli cells and spermatogenesis $)^{43}$.

Adulthood and ageing.-After puberty, in addition to their fundamental roles shown in terms of spermatogenesis ${ }^{1,44}$ and of male sexual function ${ }^{45}$, androgens have a variety of anabolic actions in the regulation of body composition, bone, muscle, glucose and lipid metabolism, erythropoiesis and cardiovascular and cognitive function over the entire lifespan ${ }^{46}$. Furthermore, several findings suggest that testosterone is neuroprotective and that declining testosterone levels during ageing are associated with cognitive and brain pathologies ${ }^{47}$. Ageing is characterized by a slow decline in testosterone levels with a substantial individual variability ${ }^{29,48-50}$; these age-related reductions in serum testosterone are mainly of primary origin in healthy men and are associated with both a loss of Leydig cells and their reduced ability to produce testosterone in response to $\mathrm{LH}^{51,52}$.

Apart from the deficiency of testosterone, hypogonadism also entails the deficiency of other testicular hormones (including INSL3, AMH and dihydrotestosterone (DHT)). As testosterone is a substrate for oestradiol synthesis, oestradiol production also decreases when testosterone levels decline. Hence, some of the symptoms associated with male hypogonadism may be due to suppressed oestrogen effects ${ }^{53}$. Among them, hypogonadal and/or ageing symptoms such as bone loss, osteoporosis, increased fat deposits and impaired sexual function may be even direct consequences of oestrogen rather than androgen deficiency.

\section{Pubertal secondary hypogonadism}

Secondary hypogonadism results from central defects of the hypothalamus or pituitary gland (BOX 2; FIG. 3).

The majority of patients with secondary hypogonadism, including those with Kallmann syndrome, respond to exogenous GnRH, pointing to a deficiency of endogenous $\mathrm{GnRH}$ as the important feature of their pathology ${ }^{54}$. A variety of LH secretory patterns can be observed in patients with congenital secondary hypogonadism. Although the majority have a complete absence of GnRH-induced LH pulses, some individuals have preserved sleepaugmented LH secretion and others demonstrate enfeebled, low-amplitude LH secretion throughout the day and night ${ }^{55}$. Although congenital secondary hypogonadism has traditionally been regarded as a permanent condition, a considerable proportion of patients $(\sim 22 \%)$ in whom the diagnosis has been confirmed with repeated biochemical assessments spontaneously 'reverse' their reproductive function ${ }^{56,57}$. These patients undergo a 
spontaneous amelioration of their disease by developing spontaneous GnRH-induced LH pulses, leading to spermatogenesis, in the absence of any fertility medications. Clinical ${ }^{58,59}$, laboratory ${ }^{60}$ and genetic ${ }^{61-63}$ characteristics of patients have been studied to identify prognostic phenotypic features for reversal, but identifying predictive characteristics has been difficult as reversal can occur even in the context of severe GnRH deficiency (that is, in those presenting with cryptorchidism, micropenis, absence of pubertal development or rare variants in a number of genes) ${ }^{57,64}$.

Genetic factors.-Over 35 loci have been implicated in the pathogenesis of congenital secondary hypogonadism. These loci can be loosely divided into two categories: genes encoding proteins that are involved in the development and migration of GnRH neurons and genes encoding proteins that are involved in the synthesis and secretion of GnRH itself (FIG 4). The first gene associated with Kallmann syndrome was ANOS1 (an X-linked gene previously called $K A L 1$ ), which encodes anosmin 1, an extracellular matrix protein that has a role in the guidance and migration of olfactory axons to the olfactory bulb ${ }^{65}$. Mutations in this gene cause a failed migration of GnRH neurons along the olfactory axons to the brain. Other genes associated with Kallmann syndrome encode proteins that work cooperatively with anosmin 1 (FIG. 4). For example, loss-of-function mutations in FGFR1 cause an autosomal dominant form of Kallmann syndrome ${ }^{65,66}$. Other genes, encoding proteins that amplify the GnRH secretory programme at puberty and modulate GnRH neuronal activity, are also associated with secondary hypogonadism by affecting GnRH secretion. Loss-offunction mutations in KISS1 and KISSIR (encoding kisspeptin 1 and its receptor, respectively) have been associated with recessive forms of secondary hypogonadism ${ }^{67,68}$. Kisspeptin is expressed in the medial basal hypothalamus and is a powerful stimulus for GnRH-induced LH secretion in mammalian species ${ }^{6,69}$. Although a powerful stimulus for GnRH secretion, kisspeptin seems to be just one part of an intricate network of hypothalamic neuropeptides that regulate GnRH release. Mutations in the genes encoding neurokinin $\mathrm{B}$ and its receptor (TAC3 and TACR3, respectively) have also been identified in patients with secondary hypogonadism ${ }^{70}$. Neurokinin B can stimulate LH secretion in some, but not all, physiological settings and may have an important role in modulating GnRH pulse frequency ${ }^{71}$.

Discovery of causal genetic variants for Kallmann syndrome led to greater understanding of the heritability of delayed puberty and the timing of puberty within the healthy population. Patients with delayed puberty are more likely than control subjects with normal pubertal timing to carry potentially pathogenetic variants in genes associated with secondary hypogonadism genes ${ }^{72}$. Moreover, delayed puberty probands seem enriched for rare variants in TAC3 or TACR 3 as compared with controls. This observation suggests that delayed puberty and secondary hypogonadism with reversal sit on the same phenotypic and genetic spectra. Further supporting the important role of neurokinin B in reproduction, a common variant near TACR3 has been shown to be associated with variation in the normal timing of female puberty ${ }^{73,74}$. 


\section{Adult hypogonadism}

Late-onset hypogonadism.-In principle, testicular function continues after puberty uninterrupted until old age. Men have no abrupt decrease in testosterone production comparable to menopause in women. However, epidemiological studies have demonstrated a $0.5-1.5 \%$ per year decrease in circulating total testosterone concentrations and a $2-3 \%$ per year decrease in free testosterone concentrations in community-dwelling middle-aged to older men beyond the age of $\sim 30$ years $27,28,75-77$. The difference between the decline of total testosterone and free testosterone over the ageing process is explained by the age-related increase in circulating concentration of sex hormone-binding globulin (SHBG), which reduces the proportion of free testosterone ${ }^{78}$. In healthy men, the age-related decline of testosterone is accompanied by an increase in $\mathrm{LH}$, indicating that it is mainly caused by a primary testicular failure compensated for by an increased LH secretion (FIG. 5). The agerelated decrease in testosterone apparently reflects general age-related cellular degeneration, reduced number of functional Leydig cells and atherosclerosis of testicular arterioles ${ }^{75}$. Nevertheless, in most men, testosterone production remains well within the reference range of young individuals, and, if there are no hypogonadal symptoms, the decline is apparently clinically nonsignificant.

Many men gain weight and acquire chronic diseases with ageing; both conditions are also associated with decreased testosterone levels ${ }^{79}$ (FIG. 5). In the EMAS, $73 \%$ of men with reduced testosterone were overweight or obese, and serum testosterone of men with a body mass index (BMI) $>30 \mathrm{~kg} / \mathrm{m}^{2}$ was $5 \mathrm{nmol} / \mathrm{l}(144 \mathrm{ng} / \mathrm{dl})$ lower than for those with normal weight ${ }^{28}$. Furthermore, a 4.5-year longitudinal arm of the EMAS ${ }^{80}$, as well as a metaanalysis ${ }^{81}$, demonstrated that weight gain suppresses and weight loss increases testosterone level. In these situations, the suppression of testosterone production is of the secondary type (that is, not accompanied by increased LH secretion but rather caused by decreased activity at the hypothalamic-pituitary level). It is also apparent that obesity (and chronic diseases) suppresses testosterone production rather than the opposite ${ }^{82}$. In real life, suppressed testosterone of ageing men emerges as the combined effect of ageing, obesity and comorbidities. Of further note, obesity mainly suppressed total testosterone, with less effect on free testosterone, because of an accompanying decrease in SHBG. Hence, symptomatic hypogonadism, apart from a biochemical reduction in total testosterone, is less common in otherwise healthy obese men ${ }^{83}$.

The exact cause of secondary hypogonadism in men with obesity with suppressed gonadotropin secretion is still not completely understood. In this context, the obesity-related suppression of HPG function has several possible mechanisms. These mechanisms include the pleiotropic inhibitory effects of adipocyte-produced adipokines, cytokines and chemokines on $\mathrm{GnRH}$ and gonadotropin secretion ${ }^{84}$ as well as obesity-related central insulin resistance ${ }^{85}$, which may negate the stimulatory effect of insulin on gonadotropin secretion. One of the candidate peptides is fat-cell-produced leptin ${ }^{86}$. Such a link is suggested by the observed decreasing effect of testosterone treatment on leptin levels in men ${ }^{87}$. Adequate leptin concentrations are needed for a normal function of the HPG axis, and in obesity cases with high leptin levels, the resistance to this hormone may explain the mechanism for its tenuous involvement in obesity-associated secondary hypogonadism ${ }^{88,89}$. The mechanisms 
of leptin resistance entail its limited access at the blood-brain barrier to the central nervous system $^{90}$, defects in leptin receptor signalling ${ }^{89}$ and hypothalamic endoplasmic reticulum stress ${ }^{91}$. Other adipokines such as the pro-inflammatory fat tissue cytokines (for example, tumor necrosis factor, IL-2 and IL-6) ${ }^{92,93}$ might also suppress gonadotropin secretion, in addition to central nervous system endocannabinoids ${ }^{94}$, central insulin resistance ${ }^{95}$ and adiponectin 96 .

By contrast, the long-held hypothesis of involvement of increased adipose tissue oestrogen production, through increased feedback inhibition of GnRH secretion, may not hold true in light of newer findings ${ }^{29}$. Serum oestrogen concentrations are in fact low in obese men ${ }^{29}$ and high only in morbidly obese men (defined by BMI $>40 \mathrm{~kg} / \mathrm{m}^{2}$ ) 97,98 . It was recently demonstrated that hyperinsulinaemia alongside elevated serum lipid levels suppresses gonadotropin secretion directly at the pituitary level, providing an additional candidate mechanism for the obesity-associated secondary hypogonadism ${ }^{99}$. Finally, the low SHBG values associated with obesity might lower the set point of the hypothalamic-pituitary feedback inhibition in relation to circulating total testosterone. Because mainly total testosterone, and to a lesser extent free testosterone, is suppressed in obese men, negative feedback inhibition of gonadotropins can be achieved at a lower level of circulating total testosterone.

\section{Diagnosis, screening and prevention \\ Diagnosis of prepubertal hypogonadism}

Disorders of sex development and treatment of neonatal hypogonadism are not the focus of this Primer; however, the dramatic changes taking place in the HPG axis during fetal and postnatal development (BOX 4) require special consideration in the diagnostic approach of male hypogonadism in later paediatric ages.

Paediatric and prepubertal hypogonadism.-After the third to sixth month of age, serum gonadotropins and testosterone normally decline (FIG. 2); thus, they are no longer informative for the diagnosis of central hypogonadism ${ }^{33}$. In this period of life, only AMH and inhibin B determinations may be helpful: low serum AMH and inhibin B in a boy with non-elevated gonadotropins point to secondary hypogonadism ${ }^{41}$ (TABLE 1). As detailed, elevated gonadotropins are indicative of primary hypogonadism. However, up to one-third of boys with complete absence of testicular tissue have normal FSH and LH in childhood, showing that primary hypogonadism is not always associated with high gonadotropin levels in childhood ${ }^{100}$.

When established during childhood, clinically evident symptoms are limited. As a result, secondary hypogonadism or primary hypogonadism may go undiagnosed unless Sertoli cell function is assessed ${ }^{101}$ (TABLE 1) Viable trisomies (chromosomal disorders characterized by additional chromosomes), such as Klinefelter syndrome and Down syndrome, are the most frequent causes of primary hypogonadism (TABLE 1). Gonadal dysfunction is present from early childhood in most boys with Down syndrome ${ }^{102}$, whereas it usually emerges during mid-puberty in patients with Klinefelter syndrome ${ }^{103}$. 
Hypogonadism in pubertal age.-Most boys have initiated pubertal development by the age of 14 years. The lack of pubertal signs by this age should prompt diagnostic evaluation (FIG. 6). In approximately two-thirds of cases, delayed puberty represents an extreme of the normal spectrum of pubertal timing (that is, CDGP) ${ }^{3}$. Family history may be informative: delayed puberty followed by spontaneous onset in a parent or sibling suggests CDGP, whereas a history of anosmia and/or hyposmia points to Kallmann syndrome ${ }^{104}$. In delayed puberty, stature is short for chronological age but adequate for bone age, which is typically delayed in CDGP. Chronic disease, medication use, inadequate nutritional status or psychosocial condition, which all can lead to functional hypogonadism ${ }^{105}$, should also be ruled out. Primary hypogonadism ${ }^{105}$ is easily diagnosed by the presence of elevated gonadotropins. On the other hand, the differential diagnosis between persistent secondary hypogonadism, responsible for $\sim 10 \%$ of cases, and CDGP may prove extremely difficult. Basal gonadotropin levels may be informative only to rule out, but not to confirm, secondary hypogonadism ${ }^{103}$, and although there is no unequivocal agreement on their usage, dynamic tests with native $\mathrm{GnRH}$ or its agonists can be considered ${ }^{106}$. A large number of different tests have been described, but none of them can ascertain the diagnosis without pitfalls because a prepubertal response may be observed in patients with CDGP in whom the HPG axis has not yet been reactivated ${ }^{105}$. It is worth mentioning that secondary hypogonadism with partial pubertal development is particularly difficult to distinguish from CDGP. The concomitant measurement of serum inhibin B may be helpful: low levels are indicative of hypogonadism whereas normal levels suggest $\mathrm{CDGP}^{107}$ (TABLE 1). Of relevance, adults with idiopathic secondary hypogonadism may have normal inhibin B levels. MRI is performed when central nervous system lesions are suspected, when delayed puberty is extreme or in patients with anosmia and/or hyposmia to demonstrate evidence of olfactorybulb aplasia and/or hypoplasia and to support the diagnosis of Kallmann syndrome ${ }^{105}$. Genetic testing is becoming more widely available and may be useful for diagnosis, prognosis ${ }^{108}$ and genetic counselling, although the utility may be limited by the variable penetrance and expression associated with many genetic causes of idiopathic secondary hypogonadism ${ }^{109}$. Comparative genomic hybridization array identifies large deletions or insertions, and candidate gene screening or next-generation sequencing are used to unveil point mutations in $>30$ genes known to be implicated in central hypogonadism following monogenic or multigenic patterns ${ }^{110}$.

\section{Diagnosis and screening in adults}

According to most international scientific societies, the mainstay of an $\mathrm{LOH}$ diagnosis includes the presence of signs and symptoms consistent with hypogonadism coupled with low morning serum testosterone levels on two or more occasions as measured with a reliable method $^{28,45,111-113}$. However, the constellation of symptoms commonly associated with $\mathrm{LOH}$ in adult men can be diverse and nonspecific and often mimics several systemic conditions, including obesity, metabolic syndrome and even the normal ageing process ${ }^{114}$. In addition, the exact cut-off values to define low testosterone levels differ depending on the society guidelines (TABLE 2). For a relatively large number of hysicians involved in the diagnosis and therapy of $\mathrm{LOH}$, as well as in the translational research dedicated to $\mathrm{LOH}$, the clinical practice guidelines first published in 2010 and recently updated by the Endocrine Society in 2018 are the most widely accepted procedures for the diagnosis and treatment of 
hypogonadism; however, adherence to these guidelines remains poor ${ }^{45,111,112}$. Thus, with a lack of a universally accepted protocol (TABLE 2), inconsistent and improper case management of LOH patients in many clinical settings has been observed ${ }^{114-116}$.

Signs and symptoms.-The symptoms of low testosterone in adult males can be difficult to diagnose. Given the myriad of pathways affected by the HPG axis and the potentially slow progression of symptoms, $\mathrm{LOH}$ signs may include a wide range of clinical presentations, including loss of libido, erectile dysfunction, depression, lethargy, anaemia and loss of muscle and bone mass ${ }^{111-113,117-119}$. At present, questionnaire-based scoring systems on LOH symptoms are not reliable enough because of their poor specificity and they are required to be followed-up with confirmatory serum testosterone testing and never used in isolation for making a diagnosis of hypogonadism ${ }^{45,113,120}$.

Serum testosterone measurements.-Testosterone measurements (taken between 7:00 and 11:00 am) in the fasting state are recommended ${ }^{45,111-113,120}$ (FIG. 1). Mass spectrometry is the gold standard of testosterone assays, but good-quality immunoassays provide fully acceptable results for clinical diagnosis ${ }^{121}$.

A lack of consensus exists regarding an absolute testosterone level indicative of $\mathrm{LOH}$ (TABLE 2). A practical definition of biochemical LOH uses cut-off concentrations of total testosterone in the range of $9.0-9.5 \mathrm{nmol} / 1(260-275 \mathrm{ng} / \mathrm{dl})$. Use of this value to define hypogonadism is supported by a randomized controlled trial (RCT) showing that testosterone treatment improves symptoms in several domains of sexual function in men with morning total testosterone $<9.1 \mathrm{nmol} / 1(262 \mathrm{ng} / \mathrm{dl})^{117}$; conversely, another trial with a total testosterone threshold of $10.4 \mathrm{nmol} / \mathrm{l}(300 \mathrm{ng} / \mathrm{dl}) \mathrm{did}$ not find improvement of sexual function $^{122}$.

The most recent update of the Endocrine Society guidelines indicates a lower limit threshold for total testosterone harmonized to the US Centers for Disease Control and Prevention standard in healthy nonobese young men of $9.2 \mathrm{nmol} / 1(264 \mathrm{ng} / \mathrm{dl})$, whereas the guidelines did not indicate any thresholds for free testosterone owing to the lack of harmonized accepted criteria ${ }^{112}$. The same authors recognized that free testosterone should be measured by an equilibrium dialysis method and that until a harmonized reference range is established, the lower limits indicated by the laboratory method may be used ${ }^{111,112}$ (TABLE 2).

According to the EMAS, the most specific diagnosis of $\mathrm{LOH}$ is reached if, in addition to total testosterone and free testosterone levels below the defined threshold concentrations (that is, $<11 \mathrm{nmol} / \mathrm{l}$ and $<220 \mathrm{pmol} / \mathrm{l}$, respectively), three sexual symptoms should be present (more specifically, erectile dysfunction, reduced morning erections and diminished sexual thoughts $)^{28}$.

If low total testosterone is observed, a second total testosterone test should be administered to confirm the diagnosis ${ }^{45,111,112}$. Clinicians should exercise caution when using total testosterone testing in men who are elderly or obese or who have diabetes as these conditions modulate the level of SHBG and, therefore, may overestimate or underestimate serum bioactive testosterone levels ${ }^{111-113,123,124}$ (FIG. 1). In these selected patients, testing for free testosterone or bioavailable testosterone is suggested, although bioavailable 
testosterone is not unanimously recommended by all guidelines ${ }^{111,112}$. In the case of free testosterone, ligand displacement immunoassays have been criticized and are currently not recommended as they are influenced by SHBG and are inaccurate ${ }^{111,112}$. The most accurate method of measuring free testosterone is equilibrium dialysis, but calculating free testosterone using total testosterone, SHBG and albumin levels and one of the algorithms (for example, the equation of Vermeulen et al. ${ }^{111,112,125}$ ) provides a sufficiently accurate estimate for clinical practice, although it has not met consensus ${ }^{126,127}$. The latest findings indicate that measurement of free testosterone (direct or calculated) improves the diagnostic accuracy of symptoms, especially in obese men ${ }^{83,113,123,128}$.

Following confirmation of low serum testosterone levels and concomitant signs and symptoms of hypogonadism ${ }^{28,45,113}$, providers should use serum LH and FSH in conjunction with testosterone to differentiate between primary and secondary hypogonadism ${ }^{29}$.

Additional analyses.-If LOH is suspected, clinicians need to comprehensively exclude acquired causes (BIX 2). Often, obesity maybe the main causative factor of $\mathrm{LOH}$. Additional laboratory tests and imaging techniques can be used to define the diagnosis. For patients suspected of primary hypogonadism, routine semen analysis and testicular sonography are examples of specialized diagnostic practices that can aid in confirming a diagnosis and localizing a lesion or specific area of testicular dysfunction ${ }^{129,130}$. Conversely, in the case of suspected secondary hypogonadism, laboratory tests such as serum prolactin levels, iron saturation and pituitary function tests can assist in identifying causes of hypothalamic and/or pituitary dysfunction. Pituitary contrast-enhanced MRI or CT may be indicated to exclude a pituitary adenoma or other lesions of the hypothalamic-pituitary region $45,111-113,130-132$. Defining the aetiology is important as it might influence management. Indeed, if, for instance, hyperprolactinaemia emerges as the cause of hypogonadism, the condition of hypogonadism needs to be differently managed using dopamine agonist therapy ${ }^{131}$.

Screening.-Screening for hypogonadism in adult men is still an area of heavy debate $^{45,111,112}$. For instance, the Endocrine Society's guidelines recommend against any form of screening in the general population ${ }^{127}$, except in patients with diabetes and bone fractures. Conversely, Endocrine Society guidelines along with the European Association of Urology (EAU), the International Society for Sexual Medicine (ISSM) and the British Society for Sexual Medicine (BSSM) guidelines suggest screening adult men with sexual dysfunction ${ }^{45,113,120}$, including those with reduced sexual desire and sexual activity, erectile dysfunction and fewer and diminished nocturnal erections (Level of Evidence (LoE) 1, grade A) ${ }^{113}$.

\section{Management}

In general, secondary hypogonadism is characterized by low or inappropriately normal gonadotropin levels; thus, the rationale is to substitute the gonadotropin deficiency with FSH and LH if fertility is desired ${ }^{133,134}$. If fertility is not an issue, testosterone therapy is advised. By contrast, when there is testicular damage, the condition is termed primary, and the only rational therapy is testosterone therapy. Testosterone therapy for congenital forms of 
hypogonadism must be lifelong; management of acquired causes depends on whether the condition is permanent or can be resolved, but testosterone treatment of $\mathrm{LOH}$ remains a matter of debate.

\section{Paediatric and pubertal hypogonadism}

The focus of this section is on the management of delayed puberty; the management of disorders of sex development and neonatal hypogonadism are reviewed elsewhere ${ }^{135,136}$. It should be noted that trials comparing different treatment regimens are lacking in delayed puberty; thus, the recommendations below are based, in part, on expert opinion and do not include all potential regimens.

CDGP.-Management of CDGP is often limited to reassurance, adult height prediction and expectant observation, particularly if puberty has started clinically and/or biochemically, but therapy with low-dose testosterone can be used as well ${ }^{104,105,137,138}$ (BOX 5). If medication is initiated, it is usually to assuage psychosocial difficulties that derive from negative interactions with peers, decreased self-esteem and anxiety about growth rate and/or body habitus.

A short course of low-dose testosterone in boys with CDGP can lead to increased growth velocity, initiation of secondary sexual characteristics and positive effects on psychosocial well-being without considerable adverse effects, rapid advancement of bone age or reduced adult height ${ }^{139,140}$. Testosterone esters given by intramuscular injection are the mainstay of treatment. A common protocol is to initiate supplementation with testosterone enanthate or cypionate each month for 3-6 months, which can be repeated for another 3-6 months with dose escalation if evidence of spontaneous puberty is lacking (BOX 5). However, intramuscular injections are painful and may require frequent health-care visits for administration. Moreover, intramuscular injections result in levels of serum testosterone that lack diurnal rhythm and are characterized by non-physiological peaks and troughs, although it is still unclear whether this is clinically significant. Hence, there is interest in alternative approaches, including use of subcutaneous testosterone injections ${ }^{141}$, oral androgens such as testosterone undecanoate ${ }^{10}$ and transdermal testosterone gels ${ }^{142,143}$, including a nasal gel that decreases the risk of person-to-person medication transfer ${ }^{144}$. Novel therapies such as synthetic kisspeptin are also being explored ${ }^{5,145}$. Although some of these agents are promising, additional data and experience are needed before these alternatives can be recommended for routine management of CDGP in place of testosterone esters.

For a subset of patients, short stature can be more concerning than delayed puberty, and CDGP is considered by some to be a subgroup of idiopathic short stature (ISS). Although growth hormones are approved for the treatment of ISS in many countries, this therapy has at best a modest effect on adult height in adolescents with CDGP and its routine use is not recommended. In boys with CDGP and short stature, another potential therapeutic approach is aromatase inhibition ${ }^{146,147}$. Aromatase inhibitors inhibit the conversion of androgens to oestrogens, the predominant hormone responsible for epiphyseal closure, raising the possibility that aromatase inhibitors could prolong growth and increase adult height. Recently, the use of aromatase inhibitors to promote endogenous puberty in boys with 
CDGP has also been reported ${ }^{148}$. However, the amount of height gained as well as the optimal timing, dose and duration of aromatase inhibitor treatment remain uncertain; moreover, potential adverse effects must be considered, and this treatment also requires further study before it should be incorporated into routine practice ${ }^{146,147}$.

Persistent hypogonadism.-In boys with persistent primary or secondary hypogonadism, initial testosterone therapy is the same as for CDGP (BOX 5), but doses are gradually increased to full adult replacement levels over $\sim 3$ years to allow development of secondary sexual characteristics. In cases of known defects, therapy can be initiated at younger ages that are more typical for pubertal initiation rather than waiting for an individual to have confirmed delayed puberty. When the differentiation of CDGP from persistent secondary hypogonadism is uncertain, time is often the defining variable; if spontaneous puberty has not occurred after 1 year of treatment, doses of testosterone can be gradually advanced towards adult levels while monitoring endogenous puberty (examination of testicular size and measurement of LH, FSH and testosterone) every 6 months.

Exogenous testosterone does not induce testicular growth or spermatogenesis, and initiation of spermatogenesis is often not possible in those with testicular defects. Induction of fertility in secondary hypogonadism requires treatment with pulsatile GnRH and/or exogenous gonadotropins (BOX 5). Over the past several years the use of gonadotropins and/or GnRH to induce puberty has garnered increasing interest. Many questions have been identified. Are fertility outcomes better if FSH is administered before administration of human chorionic gonadotropin (hCG)? Would FSH pretreatment versus GnRH administration alone improve outcomes, especially for those with the smallest testes? Is $\mathrm{GnRH}$ administration superior to gonadotropin therapy? Perhaps most important for adolescents is the question of whether fertility outcomes would be improved if GnRH and/or gonadotropins were used to induce puberty instead of waiting to administer these agents in adulthood ${ }^{104,137,138,149,150}$. For the last question, more research is needed before such therapies are routinely recommended for pubertal induction in place of the less invasive and less expensive use of testosterone.

Regardless of the treatment regimen used, it is important that the care of these patients be transferred successfully from paediatric care to adult care providers.

\section{Hypogonadism in adults}

The management of genetic secondary hypogonadism depends on the type of the underlying disease and on patients' needs. In adulthood, when fertility is required, the most widely used compound is injected hCG (intramuscular or subcutaneously) weekly, alone or in combination with FSH weekly. In men, the recombinant preparations seem not to offer significant advantages when compared with the purified compounds, derived from the urine of either pregnant or postmenopausal women ${ }^{151}$. Fertility can also be induced using GnRH administered in a pulsatile manner, but this treatment is not widely available ${ }^{151}$. Conversely, the use of anti-oestrogens is useless and even contraindicated in these individuals ${ }^{133}$.

Men with genetic disorders.-In males with Klinefelter syndrome, the degree of androgen deficiency depends on the number and residual function of Leydig cells; spontaneous fertility is rarely observed ${ }^{152}$. Likewise, even among men with Klinefelter 
syndrome, hypogonadism is associated with greater risk of metabolic syndrome, T2DM, cardiovascular disease (CVD), breast cancer and extragonadal germ cell tumours ${ }^{25}$.

Testosterone replacement therapy is the mainstay of treatment in hypogonadal patients with Klinefelter syndrome; however, randomized trials are needed to determine optimal therapeutic regimens and subsequent follow-up schedules ${ }^{22}$. Although the degree of virilization as well as the levels of testosterone are usually normal at puberty in boys with Klinefelter syndrome, some evidence has suggested that an early treatment with testosterone should be offered to the majority of patients, starting from the peri-pubertal period, to ensure an optimal development of sexual characteristics, muscle bulk and bone structure and to prevent the long-term negative consequences of hypogonadism, including metabolic diseases and mental impairment ${ }^{153}$. However, available data on this topic are conflicting and are largely based on nonrandomized studies. An age-dependent decline in testosterone is reported with a high prevalence of hypogonadism detected in adulthood ${ }^{154,155}$. Hence, testosterone therapy in Klinefelter syndrome should be offered in the presence of documented reduced levels of total testosterone $(<12 \mathrm{nmol} / 1(<3.5 \mathrm{ng} / \mathrm{ml}))$ associated with symptoms, in adulthood, or earlier as a consequence of delayed puberty ${ }^{152,153}$.

Infertility in men with Klinefelter syndrome had long been considered an untreatable condition. Recent data have emphasized that individuals with Klinefelter syndrome may benefit from assisted reproductive techniques owing to the presence of residual foci of preserved spermatogenesis within the testis. Accordingly, a recent meta-analysis of the available data has documented that testicular sperm extraction can be successful in almost $50 \%$ of individuals with Klinefelter syndrome, with a subsequent live birth rate close to $50 \% 156$. All these results seem to be independent of any clinical or biochemical parameters tested $^{156}$ or the age at which surgery was performed ${ }^{157}$.

Late-onset hypogonadism.-In contrast to testosterone therapy for congenital forms of hypogonadism, which has to continue throughout the lifespan of the man, testosterone treatment of LOH is controversial because of unclear indications for replacement and potential risks in older individuals that have been widely and often harshly debated without a definitive conclusion ${ }^{45,158}$. Ageing men may develop low testosterone mainly owing to being overweight, inactive and having chronic diseases ${ }^{28,45}$. The first advice for these patients is to address modifiable risk factors - if present — and to improve lifestyle through exercise, reducing weight and good treatment balance of comorbidities ${ }^{80,159-161}$. These modifications may result in serum testosterone level improvement (although it may not become fully normalized) and reduce associated symptoms and health risks ${ }^{159}$. Moreover, in real life, lifestyle modification may be difficult; thus, the pharmacological reversal of reduced testosterone levels has been promoted as an alternative. Indeed, low testosterone is a biomarker for impaired general health, and treatment of comorbid conditions is important in combination with testosterone therapy $45,46,49,162$. Overall, it is necessary to underline how the debate related to testosterone therapy in men with clinical and biochemical characteristics suggestive of $\mathrm{LOH}$ is still continuing. In this context, the principle of testosterone therapy is based on the assumption that low testosterone levels are the cause (that is, risk factor) of the hypogonadism-associated symptoms rather than their consequence. Although the causality is definitely bidirectional, evidence is mounting that 
low testosterone is primarily the consequence (that is, risk marker) of ill health rather than its cause ${ }^{80,81}$. Thus, testosterone therapy, aimed at treating symptoms associated with low testosterone and improving quality of life in $\mathrm{LOH}$, remains controversial without a definitive and unanimously accepted conclusion ${ }^{120}$. Indeed, although improvement of symptoms is often observed, unclear indications for replacement, the optimum serum testosterone levels and potential risks (especially cardiovascular risk) in older individuals have led to conflicting recommendations by societies, as outlined below. The most worrying gap in our knowledge at the moment is the paucity of information on potential long-term effects, both positive and negative. To this aim, we also discuss recommendations in terms of follow-up and contraindications for treatment.

As detailed, common symptoms in men with low testosterone are sexual dysfunction; less specific conditions associated with low testosterone, including unexplained anaemia, osteoporosis, loss of vigour, frailty, insomnia, cognitive dysfunction and depression, have been described, although these symptoms may occur also in older men with normal serum testosterone levels and on their own seldom justify the diagnosis of $\mathrm{LOH}^{120}$. Recently, RCTs have advanced our knowledge on indications for testosterone therapy $45,113,117,120,163$ : sexual symptoms may improve, including erectile dysfunction and loss of libido. For patients with T2DM and erectile dysfunction, this was only the case in men with clearly reduced testosterone levels $(<8 \mathrm{mmol} / 1(2.31 \mathrm{ng} / \mathrm{ml}))^{164}$. From a pathophysiological standpoint, it has been argued that this is because erectile dysfunction in T2DM is predominantly due to vascular and neuropathic disease and is, therefore, not likely to be the case in those men who do not have an established vascular disease.

The Birmingham, Lichfield, Atherstone, Sutton Coldfield, and Tamworth (BLAST) RCT showed that the administration of injectable testosterone undecanoate or placebo for 30 weeks to hypogonadal men with T2DM promoted a significant reduction in glycated haemoglobin $\left(\mathrm{HbA}_{1 \mathrm{c}}\right)$ in testosterone-treated patients compared with placebo-treated men at 6 weeks and 18 weeks, but not at 30 weeks ${ }^{165}$. Among non-depressed men, those treated with testosterone undecanoate had significant improvements in BMI, weight, waist circumference, erectile function scores and Aging Males' Symptoms (AMS) scores ${ }^{165}$. The Testosterone Replacement in Hypogonadal Men with Type 2 Diabetes and/or Metabolic Syndrome (TIMES2) trial evaluated symptomatic hypogonadal men treated with testosterone gel or placebo for 12 months ${ }^{166}$. At 6 months, testosterone-treated patients had reduced insulin resistance (assessed by the homeostasis model assessment of insulin resistance (HOMA-IR)) compared with placebo-treated patients, reduced lipo-protein A (Lpa), reduced high-density lipoprotein (HDL) and improved erectile function scores, although no improvements of $\mathrm{HbA}_{\mathrm{lc}}$ levels were observed. Among participants with metabolic syndrome, testosterone therapy reduced Lpa and low-density lipoprotein (LDL) compared with placebo ${ }^{166}$. Hence, although testosterone treatment of men with LOH has in some studies shown modest improvement in glycaemic control, such data overall are inconsistent. The current evidence is insufficient to support testosterone therapy to improve the metabolic status in older men with $\mathrm{LOH}$ or in particular those with T2DM or the metabolic syndrome. The recent Endocrine Society guidelines support this conclusion ${ }^{112}$. 
One of the hallmarks of male hypogonadism is osteoporosis. Testosterone treatment of hypogonadal men has been shown to bring about variable increases in areal and volumetric bone mineral density in lumbar, spinal, vertebral and femoral neck regions ${ }^{167-169}$. In general, the magnitude of the treatment effect is inversely related to basal serum testosterone ${ }^{118}$. Unfortunately, information about treatment response of the most important clinical end point (that is, fracture prevention) is completely missing. Expert opinions consider the specific anti-resorptive treatments and osteoanabolic agents effective in preventing bone loss in hypogonadal men ${ }^{170,171}$. Testosterone treatment as monotherapy for osteoporosis is recommended only in hypogonadal men in whom there are contraindications for approved anti-resorptive therapies. Nevertheless, testosterone may have a modest positive effect on bone health in men whose testosterone treatment has been initiated for other reasons.

An additional common sign of hypogonadism in ageing men is unexplained anaemia. Convincing data exist that this symptom responds favourably to testosterone treatment ${ }^{172,173}$, even to the extent that polycythaemia is a common adverse effect of the therapy and must be carefully controlled. However, in the Testosterone Trials (TTrials), the average increase in haemoglobin by $10 \mathrm{~g} / \mathrm{l}$ was not found to be associated with clinically significant improvements of physical performance ${ }^{173}$.

Numerous studies also suggested an association between serum testosterone levels and depressive symptoms ${ }^{174}$. However, the relationship between low testosterone and depression seems to be complex and associated with many factors, such as androgen receptor genetic polymorphisms ${ }^{175,176}$. A registry study of 762 hypogonadal men found that $92.4 \%$ of men demonstrated some level of depressive symptoms, with $17.3 \%$ of men having moderately severe to severe depressive symptoms ${ }^{174}$. After 12 months of testosterone therapy, the percentage of patients with moderately severe to severe symptoms decreased from $17.3 \%$ to $2.1 \%{ }^{174}$. In the Vitality Trial, there were significantly greater improvements between the testosterone therapy group and the placebo group in the 36-Item Short-Form Health Survey (SF-36) vitality score (mean difference 2.41 points; $P=0.03$ ) and the Patient Health Questionnaire 9 (PHQ-9) depression score (mean difference -0.72 points; $P=0.004)^{117}$. More recently, findings from a meta-analysis aimed at assessing the association of testosterone treatment with depressive symptoms in men showed a moderate antidepressant association of testosterone treatment translatable into a clinically relevant symptom reduction ${ }^{119}$. This effect was confirmed only in men with hypogonadism and in those having more subtle symptoms of depression. However, the large portion of studies with high or unclear risk of bias and the low number of methodologically rigorous RCTs primarily addressing the effect of testosterone treatment in depressed but otherwise healthy men limit the interpretation, as per the admission of the authors. Considering all available information, testosterone treatment of men with LOH may have a slight improving effect on mood, but there is no convincing evidence suggesting that testosterone therapy could be used to treat depression of older men. Other mental well-being symptoms of LOH, such as cognitive dysfunction, did not significantly improve under testosterone therapy compared with placebo $^{177}$. 
Recently, findings from the Physical Function Trial, one of the of seven TTrials, demonstrated that testosterone therapy consistently improved self-reported walking ability in men $>65$ years of age and modestly improved 6-minute walk test distance but did not affect falls ${ }^{178}$. The effect of testosterone on mobility measures was related to baseline gait speed and self-reported mobility limitation and changes in testosterone and haemoglobin concentrations $^{178}$.

Although there are as yet insufficient data to define optimal serum levels of testosterone during testosterone therapy, the aim is to restore serum testosterone to physiological levels ${ }^{45,112,113,120}$, and most of the scientific societies suggest they aim at achieving testosterone concentrations in the mid-normal range during treatment with any of the approved formulations, usually taking into consideration the patient's preference as the criterion of choice of preparation after having involved the patient in decision-making and the potential benefits and risks of therapy have been discussed ${ }^{45,112,113,120}$. Several applications of testosterone are available for testosterone therapy, induding oral preparations, transdermal gels and intramuscular injections. Truly comparative studies of different applications are not available ${ }^{45,120}$.

Testosterone therapy is associated with a number of possible adverse events, including an increase in red blood cells and an elevation of prostate-specific antigen (PSA) ${ }^{112}$. Although observational studies do not indicate a higher risk of thrombosis or prostate cancer in men undergoing testosterone therapy, monitoring remains highly advisable ${ }^{112,113,120,179,180}$. As a whole, serious adverse events related to testosterone therapy are relatively rare. Overall, they are more significant in elderly patients and are often dependent on the method of delivery. Some adverse events are related to supraphysiologic levels and can be lowered or stopped altogether by adjusting the dose or switching to a different formulation ${ }^{113}$.

In terms of treatment follow-up, it is strongly recommended to perform haematological, cardiovascular, breast and prostatic assessment before the start of treatment ${ }^{45,112,113,120,179,180}$. After testosterone therapy has been established, it is suggested to assess the response to testosterone treatment at 3 months, 6 months and 12 months after the onset of treatment and thereafter annually $45,112,113,120,179,180$. This assessment includes monitoring haematocrit (haematocrit levels should remain $<54 \%$ ), haemoglobin, testosterone values and PSA during testosterone treatment. Men with CVDs should be monitored carefully throughout the follow-up $45,112,113,120,179,180$. Decisions to continue treatment should be based not only on avoiding adverse events but also on a documented improvement in hypogonadism symptoms. In fact, owing to the aspecificity of $\mathrm{LOH}$ clinical features, their improvement upon testosterone therapy is useful information to corroborate the initial diagnosis and a reason to continue treatment if adverse events do not occur. As suggested by the Endocrine Society guidelines ${ }^{112}$, symptom monitoring should be performed at 3 months after treatment initiation and then annually.

Contraindications for testosterone therapy are locally advanced and metastatic prostate cancer. In addition, breast cancer, although rare, is considered a contraindication for this therapy $45,112,113,120$. Likewise, men with an active wish for children should not use 
exogenous testosterone because it inhibits spermatogenesis owing to suppression of gonadotropin secretion and intratesticular testosterone $45,112,120,181$.

Some evidence suggests that hypogonadal men have an increased risk of CVD ${ }^{182}$. In this context, a recent meta-analysis, including 37 observational studies published between 1988 and 2017 and enrolling 43,041 men with a mean follow-up of 333 weeks, showed that low endogenous testosterone at enrolment predicted overall and cardiovascular mortality and cardiovascular morbidity ${ }^{183}$. With normalization of serum testosterone levels to the physiological range, this risk could theoretically decrease, but the causality between low testosterone and CVD risk remains unclear. Indeed, many observational studies indicated that the normalization of serum testosterone levels to the physiological range can improve metabolic risk factors (such as obesity, diabetes and metabolic syndrome) ${ }^{184,185}$.

Conversely, data from RCTs are still conflicting and not unequivocal ${ }^{186}$. Moreover, evidence mostly obtained from observational studies has suggested a possible increased CVD risk related to testosterone therapy ${ }^{187}$. The CVD risk seems to be higher at the beginning of the treatment ${ }^{188}$. As a sign of conflict and confusion about the position of testosterone treatment of LOH, the US FDA cautioned that the benefits and safety of testosterone replacement therapy have not been clearly established for the treatment of low testosterone levels in older men $^{189}$. This position has been endorsed by Health Canada ${ }^{190}$ and by the Australian Society of Endocrinology ${ }^{191}$. By contrast, the European Medicines Agency (EMA), after its own review of the available data, did not find sufficient evidence for declaring a testosterone replacement therapy-associated cardiovascular risk ${ }^{192}$. In line with this position, a recent meta-analysis including 15 pharmaco-epidemiological trials and 93 RCTs evaluating the cardiovascular safety of testosterone replacement treatment concluded that when testosterone treatment is correctly applied, it is not associated with an increase in CVD risk $^{193}$. Conversely, the same study documented that an increased cardiovascular risk is observed when testosterone treatment is used at dosages higher than those routinely recommended ${ }^{193}$. Debate is still ongoing, and the potential risks of CVD and venous thromboembolism associated with testosterone therapy in older men with symptomatic low serum testosterone may not be resolved until results of adequately powered RCTs specifically designed to this end are available. Such a study (TRAVERSE), sponsored by several major drug companies, has recently been initiated in the United States (). Further confusion to this issue was added by a very recent Mendelian randomization study from the United Kingdom, reporting that the genetically predicted endogenous testosterone levels were positively associated with thromboembolism, heart failure and myocardial infarction in men ${ }^{194}$.

Development of polycythaemia during testosterone treatment is relatively common, with frequency ranging from $2 \%$ to $7 \%$ and older men appearing to be at higher risk ${ }^{195}$. Thus, elevated haematocrit is considered a contraindication of testosterone treatment, and adherence to the guidelines advising frequent follow-up of haemoglobin or haematocrit is important. 
Finally, testosterone treatment has been documented to worsen the symptoms of obstructive sleep apnoea ${ }^{196}$, and severe untreated sleep apnoea is included in the contraindications of testosterone treatment in ageing men ${ }^{112}$.

\section{Quality of life}

As previously detailed, the EMAS found that the presence of at least three sexual symptoms offered the greatest sensitivity and specificity in identifying hypogonadal patients ${ }^{28}$. The association between these sexual symptoms and low testosterone was statistically significant (OR 1.71; CI 1.08-2.63) and remained significant after adjustment for age, BMI and coexisting illnesses. As discussed in prior sections, sexual dysfunction symptoms are probably the symptoms most consistently associated with low serum testosterone in elderly men ${ }^{197-199}$. In this context, a number of meta-analyses demonstrated significant improvements in overall erectile function in hypogonadal men initiating testosterone therapy. Isidori et al. ${ }^{200}$, for instance, evaluated 17 placebo-controlled, RCTs of testosterone therapy in hypogonadal and eugonadal men. Among those men with low serum testosterone levels $(<10 \mathrm{nmol} / 1(<288 \mathrm{ng} / \mathrm{dl}))$, there were significant improvements with testosterone therapy in morning erections, sexual motivation, erectile function, sexual thoughts, sexual satisfaction, episodes of successful intercourse and total erections and/or ejaculations ${ }^{200}$. Recently, Corona et al. ${ }^{197}$ conducted a meta-analysis of RCTs addressing the effects of testosterone therapy to treat erectile dysfunction with a mean follow-up of 40.1 weeks ${ }^{197}$. The results demonstrated that testosterone therapy significantly improved erectile function compared with placebo (International Index of Erectile Function-Erectile Function domain (IIEF-EF) mean difference $=2.31(95 \%$ CI 1.41-3.22)). Patients with more severe hypogonadism reported greater improvements in erectile function compared with those with milder testosterone deficiency; in this context, the authors found that those men with a serum testosterone level $<8 \mathrm{nmol} / 1(231 \mathrm{ng} / \mathrm{dl})$ had the greatest improvement in erectile function following testosterone therapy. According to the TTrial, designed to determine the efficacy of testosterone therapy in older hypogonadal men ( $\Varangle 65$ years of age $)^{117}$, testosterone therapy was also associated with increased sexual desire according to the Derogatis Interview for Sexual Functioning in Men-II (DISF-M-II) (treatment effect 2.93; $P<0.001$ ) and increased erectile function according to the IIEF (treatment effect 2.64; $P<0.001$ ). More precisely, $20 \%$ of men treated with testosterone reported that their sexual desire was much better since the beginning of the trial $(P<0.001)$, as compared with $<10 \%$ of those individuals who received placebo instead ${ }^{117}$. One weakness of the TTrials is their short duration (1 year), and it remains unknown whether more promising results could be obtained upon longer treatment. Conspicuously, another similar RCT on slightly younger men (TEAAM) showed no improvement in sexual function during 3 years of testosterone treatment ${ }^{122}$. Thus, we can conclude that testosterone therapy may improve several aspects of sexual function, but only in men with clearly hypogonadal concentrations of testosterone.

Finally, uncontrolled trials have demonstrated that hypogonadal patients not responding to PDE5 inhibitors (PDE5Is) may improve their response to PDE5Is after initiating testosterone therapy ${ }^{201-203}$. In the real-world setting, most patients with erectile dysfunction will first be prescribed a PDE5I, which is usually effective ${ }^{201}$; however, if diagnostic criteria 
suggestive for testosterone deficiency are present, testosterone therapy is the more appropriate treatment even in erectile dysfunction patients.

\section{Outlook}

\section{Klinefelter syndrome}

A longstanding question has been how the presence of an additional $\mathrm{X}$ chromosome leads to the various features of Klinefelter syndrome. Big data approaches have been used to identify new clinical associations with Klinefelter syndrome ${ }^{204}$, and transcriptomic, epigenomic, proteomic and metabolomic approaches have the potential to identify specific pathways affected by Klinefelter syndrome ${ }^{205-208}$. These studies may lead to the development of targeted treatments for the many issues associated with Klinefelter syndrome.

When to start androgen treatment in individuals with Klinefelter syndrome is an area of active investigation. Whereas frank hypogonadism does not typically become evident until late adolescence or adulthood, it has been proposed that boys with Klinefelter syndrome have some degree of testosterone deficiency throughout life and that this testosterone deficiency may contribute to differences in body composition, learning and behavioural challenges and increased risk of CVD. In this context, some studies suggested that early testosterone therapy might be beneficial for individuals with Klinefelter syndrome. A retrospective analysis including 101 boys with Klinefelter syndrome and micropenis showed that early testosterone treatment (from early infancy) improved neuromotor function, speech and language, and intellectual and reading function ${ }^{209}$.

It is important to recognize that the evidence for these effects has largely come from observational studies, but randomized trials of early testosterone therapy are now underway ().

\section{Diagnosis}

Currently, the only way to definitively determine whether a child has self-limited CDGP or more persistent idiopathic secondary hypogonadism is to monitor over time to determine whether the child eventually enters puberty and achieves normal adult reproductive endocrine function or not (). Because CDGP is the more common diagnosis, the initial approach to management is typically reassurance and watchful waiting, with treatment with sex steroids deferred until well after the typical age for entering puberty. A prospective method to distinguish constitutional delay from idiopathic secondary hypogonadism would allow those with idiopathic secondary hypogonadism, for whom reassurance is inappropriate, to receive more timely treatment. However, despite decades of searching, such a method does not currently exist ${ }^{210}$.

Building on the identification of both rare and common genetic variants that contribute to constitutional delay and idiopathic secondary hypogonadism, genetic testing may lead to earlier diagnosis ${ }^{211}$. However, this approach may be complicated by genetic overlap between these two conditions, and variable penetrance and expressivity may limit the predictive power of genetic testing ${ }^{72}$. 
Provocative stimulation tests offer another potential method to assess future reproductive endocrine potential in prepubertal children. Stimulation tests using hCG, GnRH or GnRH analogues have been found to lack complete sensitivity or specificity ${ }^{210}$, but stimulation testing using recently identified factors that function upstream of the GnRH neuron, such as kisspeptin, may hold promise ${ }^{212}$.

\section{Treatment}

For decades, testosterone therapy has been used to induce pubertal changes in children with secondary hypogonadism, with gonadotropin treatment typically reserved for when fertility is desired. Recently, there has been renewed interest in the use of gonadotropins as a more physiologic method for pubertal induction, as gonadotropin treatment induces testicular growth and spermatogenesis in addition to testosterone production in boys ${ }^{104,137,149,213}$. Gonadotropin treatment has also been used during the mini-puberty of infancy ${ }^{214,215}$. Trials directly comparing testosterone to gonadotropin treatment will determine whether the benefits of gonadotropin treatment justify the substantially higher cost.

\section{Adults and ageing men}

In 'functional' hypogonadism (BOX 1), PDE5Is are first-line pharmacotherapy to improve erectile dysfunction ${ }^{31}$. Testosterone therapy can be considered if the previous strategies fail. Lifestyle change is also strongly recommended in patients with T2DM, a condition with a similar prevalence to hypogonadism, characterized by vague symptoms, including sexual dysfunction ${ }^{216}$. In T2DM, in the presence of defined glucose abnormalities - along with lifestyle changes - medical therapy is strongly recommended, although in rare cases an 'organic' alteration is found (1\%). Evidence from RCTs indicates that testosterone therapy in 'functional' hypogonadism is able to improve sexual dysfunction ${ }^{200}$ and body composition $^{186}$. In addition, meta-analyses of RCTs did not support an association between testosterone therapy and an increased cardiovascular risk ${ }^{217-224}$. One of the main limitations in interpreting data from available RCTs is that all currently published controlled studies were conducted for a relatively short period of no more than 3 years. Hence, information concerning advantages and disadvantages of testosterone medications throughout a longer period of time is not yet available. In particular, we need larger and sufficiently powered studies with longer duration, and with specific aims, to better clarify hard treatment end points. In this framework, multicentre international register studies are welcome ().

\section{References}

1. Corradi PF, Corradi RB \& Greene LW Physiology of the hypothalamic pituitary gonadal axis in the male. Urol. Clin. North Am 43, 151-162 (2016). [PubMed: 27132572] This manuscript comprehensively describes the complex physiology of the male HPG axis.

2. Fluck CE et al. Why boys will be boys: two pathways of fetal testicular androgen biosynthesis are needed for male sexual differentiation. Am. J. Hum. Genet 89, 201-218 (2011). [PubMed: 21802064]

3. Ross A \& Bhasin S Hypogonadism: its prevalence and diagnosis. Urol. Clin. North Am 43, 163-176 (2016). [PubMed: 27132573]

4. Rey RA et al. Male hypogonadism: an extended classification based on a developmental, endocrine physiology-based approach. Andrology 1,3-16 (2013). [PubMed: 23258624] This work provides a classification of male hypogonadism, explaining the pathophysiology and specific diagnostic 
procedures needed according to the age of establishment of the disorder, from fetal life to adulthood.

5. Rastrelli G, Vignozzi L \& Maggi M Different medications for hypogonadotropic hypogonadism. Endocr. Dev 30, 60-78 (2016). [PubMed: 26683738]

6. Lippincott MF et al. Kisspeptin responsiveness signals emergence of reproductive endocrine activity: implications for human puberty. J. Clin. Endocrinol. Metab 101, 3061-3069 (2016). [PubMed: 27214398]

7. Hughes IA, Nihoul-Fékété C, Thomas B \& Cohen-Kettenis PT Consequences of the ESPE/LWPES guidelines for diagnosis and treatment of disorders of sex development. Best Pract. Res. Clin. Endocrinol. Metab 21, 351-365 (2007). [PubMed: 17875484]

8. Maggi M \& Buvat J Standard operating procedures: pubertas tarda/delayed puberty-male. J. Sex. Med 10, 285-293 (2013). [PubMed: 22376050]

9. Sedlmeyer IL Delayed puberty: analysis of a large case series from an academic center. J. Clin. Endocrinol. Metab 87, 1613-1620 (2002). [PubMed: 11932291]

10. Lawaetz JG et al. Evaluation of 451 Danish boys with delayed puberty: diagnostic use of a new puberty nomogram and effects of oral testosterone therapy. J. Clin. Endocrinol. Metab 100, 13761385 (2015). [PubMed: 25594861]

11. Varimo I, Miettinen PJ, Känsäkoski J, Raivio T \& Hero M Congenital hypogonadotropic hypogonadism, functional hypogonadotropism or constitutional delay of growth and puberty? An analysis of a large patient series from a single tertiary center. Hum. Reprod 32, 147-153 (2016). [PubMed: 27927844]

12. Abitbol L, Zborovski S \& Palmert MR Evaluation of delayed puberty: what diagnostic tests should be performed in the seemingly otherwise well adolescent? Arch. Dis. Child 101, 767-771 (2016). [PubMed: 27190100]

13. Piel FB, Steinberg MH \& Rees DC Sickle cell disease. N. Engl. J. Med 376, 1561-1573 (2017). [PubMed: 28423290]

14. Kupczyk M \& Wenzel S US and European severe asthma cohorts: what can they teach us about severe asthma? J. Intern. Med 272, 121-132 (2012). [PubMed: 22630041]

15. Cosnes J, Gower-Rousseau C, Seksik P \& Cortot A Epidemiology and natural history of inflammatory bowel diseases Gastroenterology 140,1785-1794 (2011). [PubMed: 21530745]

16. Benchimol EI et al. Epidemiology of pediatric inflammatory bowel disease: a systematic review of international trends. Inflamm. Bowel Dis. 17, 423-439 (2011). [PubMed: 20564651]

17. Bonomi $\mathrm{M}$ et al. Characteristics of a nationwide cohort of patients presenting with isolated hypogonadotropic hypogonadism (1HH). Eur. J. Endocrinol 178, 23-32 (2018). [PubMed: 28882981]

18. Franco B et al. A gene deleted in Kallmann's syndrome shares homology with neural cell adhesion and axonal path-finding molecules. Nature 353, 529-536 (1991). [PubMed: 1922361]

19. Guyot-Goubin A et al. Descriptive epidemiology of childhood Langerhans cell histiocytosis in France, 2000-2004. Pediatr. Blood Cancer 51, 71-75 (2008). [PubMed: 18260117]

20. Bakhsheshian $\mathrm{J}$ et al. Risk factors associated with the surgical management of craniopharyngiomas in pediatric patients: analysis of 1961 patients from a national registry database. Neurosurg. Focus 41, E8 (2016).

21. Bonomi M et al. Klinefelter syndrome (KS): genetics, clinical phenotype and hypogonadism. J. Endocrinol, invest 40, 123-134 (2016). [PubMed: 27644703]

22. Kanakis GA \& Nieschlag E Klinefelter syndrome: more than hypogonadism. Metabolism 86, 135144 (2018). [PubMed: 29382506]

23. Aksglaede L et al. 47,XXY Klinefelter syndrome: Clinical characteristics and age-specific recommendations for medical management. Am. J. Med. Genet C 163, 55-63 (2013).

24. Bojesen A, Juul S \& Gravholt CH Prenatal and postnatal prevalence of Klinefelter syndrome: a national registry study. J. Clin. Endocrinol. Metab 88, 622-626 (2003). [PubMed: 12574191]

25. Gravholt $\mathrm{CH}$ et al. Klinefelter syndrome: integrating genetics, neuropsychology, and endocrinology. Endocr. Rev 39, 389-423 (2018). [PubMed: 29438472] 
26. Leader A, Lishner M, Michaeli J \& Revel A Fertility considerations and preservation in haematooncology patients undergoing treatment. Br. J. Haematol 153, 291-308 (2011). [PubMed: 21391973]

27. Harman SM et al. Longitudinal effects of aging on serum total and free testosterone levels in healthy men. J. Clin. Endocrinol. Metab 86, 724-731 (2001). [PubMed: 11158037]

28. Wu FCW et al. Identification of late-onset hypogonadism in middle-aged and elderly men. N. Engl. J. Med 363, 123-135 (2010). [PubMed: 20554979] This study defines the diagnostic criteria and prevalence of symptomatic $\mathrm{LOH}$ in the European male population.

29. Tajar A et al. Characteristics of secondary, primary, and compensated hypogonadism in aging men: evidence from the European Male Ageing Study. J. Clin. Endocrinol. Metab 95, 1810-1818 (2010). [PubMed: 20173018]

30. Eendebak RJAH et al. Elevated luteinizing hormone despite normal testosterone levels in older men-natural history, risk factors and clinical features. Clin. Endocrinol 88, 479-490 (2018).

31. Grossmann M \& Matsumoto AM A Perspective on middle-aged and older men with functional hypogonadism: focus on holistic management. J. Clin. Endocrinol. Metab 102, 1067-1075 (2017). [PubMed: 28359097] This study launches the new concept of functional hypogonadism.

32. Maseroli E et al. Prevalence of endocrine and metabolic disorders in subjects with erectile dysfunction: a comparative study. J. Sex. Med 12, 956-965 (2015). [PubMed: 25689116]

33. Kuiri-Hänninen T, Sankilampi U \& Dunkel L Activation of the hypothalamic-pituitary-gonadal axis in infancy: minipuberty. Horm. Res. Paediatr 82, 73-80 (2014). [PubMed: 25012863] This paper reviews the physiology and pathophysiology of disorders occurring during the postnatal activation of the gonadal axis.

34. Bergadá I et al. Time course of the serum gonadotropin surge, inhibins, and anti-Müllerian hormone in normal newborn males during the first month of life. J. Clin. Endocrinol. Metab 91, 4092-4098 (2006). [PubMed: 16849404]

35. Grinspon RP et al. Male central precocious puberty: serum profile of anti-Müllerian hormone and inhibin B before, during, and after treatment with GnRH analogue Int. J. Endocrinol 2013, 823064 (2013). [PubMed: 24324495]

36. Dunkel L \& Quinton R Transition in endocrinology: induction of puberty. Eur. J. Endocrinol 170, R229-R239 (2014). [PubMed: 24836550]

37. Mouritsen A et al. The pubertal transition in 179 healthy Danish children: associations between pubarche, adrenarche, gonadarche, and body composition. Eur. J. Endocrinol 168, 129-136 (2013). [PubMed: 23093700]

38. Nathan BM \& Palmert MR Regulation and disorders of pubertal timing. Endocrinol. Metab. Clin. North Am 34, 617-641 (2005). [PubMed: 16085163] This paper describes the physiology and pathophysiology of disorders occurring during the pubertal timing.

39. Marshall WA \& Tanner JM Variations in pattern of pubertal changes in girls. Arch. Dis. Child 44, 291-303 (1969). [PubMed: 5785179]

40. Marshall WA \& Tanner JM Variations in the pattern of pubertal changes in boys. Arch. Dis. Child 45, 13-23 (1970). [PubMed: 5440182] This manuscript is still a milestone in the field.

41. Grinspon RP et al. Spreading the clinical window for diagnosing fetal-onset hypogonadism in boys. Front. Endocrinol. 5, 51 (2014).

42. Grinspon RP, Urrutia M \& Rey RA Male central hypogonadism in paediatrics - the relevance of follicle-stimulating hormone and Sertoli cell markers. Eur. Endocrinol 14, 67 (2018). [PubMed: 30349597]

43. Andersson AM \& Skakkebaek NE Serum inhibin B levels during male childhood and puberty. Mol. Cell. Endocrinol 180, 103-107 (2001). [PubMed: 11451578]

44. Neto FTL, Bach PV, Najari BB, Li PS \& Goldstein M Spermatogenesis in humans and its affecting factors. Semin. Cell Dev. Biol 59, 10-26 (2016). [PubMed: 27143445]

45. Khera $\mathrm{M}$ et al. Diagnosis and treatment of testosterone deficiency: recommendations from the Fourth International Consultation for Sexual Medicine (ICSM 2015). J. Sex. Med 13,1787-1804 (2016). [PubMed: 27914560] This manuscript mostly deals with the paramount role of decreasing testosterone levels in terms of male sexual function impairment according to both the literature and the opinion of a panel of experts in the field of sexual medicine.

Nat Rev Dis Primers. Author manuscript; available in PMC 2020 January 06. 
46. Swiecicka A et al. Reproductive hormone levels predict changes in frailty status in communitydwelling older men: European Male Ageing Study Prospective Data. J. Clin. Endocrinol. Metab 103, 701-709 (2017).

47. Yeap BB Hormonal changes and their impact on cognition and mental health of ageing men. Maturitas 79, 227-235 (2014). [PubMed: 24953176]

48. Hsu B et al. Temporal trend in androgen status and androgen-sensitive outcomes in older men. J. Clin. Endocrinol. Metab 101, 1836-1846 (2016). [PubMed: 26918290]

49. Holmboe SA et al. Individual testosterone decline and future mortality risk in men. Eur J. Endocrinol 178, 121-128 (2018).

50. Travison TG et al. Harmonized reference ranges for circulating testosterone levels in men of four cohort studies in the United States and Europe. J. Clin. Endocrinol. Metab 102, 1161-1173 (2017). [PubMed: 28324103] This study is the first to detail reference ranges for serum testosterone concentrations throughout the male age span.

51. Wang Y, Chen F, Ye L, Zirkin B \& Chen H Steroidogenesis in Leydig cells: effects of aging and environmental factors. Reproduction 154, R111-R122 (2017). [PubMed: 28747539]

52. Neaves WB, Johnson L, Proter JC, Parker CR Jr \& Petty CS Leydig cell numbers, daily sperm production, and serum gonadotropin levels in aging men. J. Clin. Endocrinol. Metab 59, 756-763 (1984). [PubMed: 6434579]

53. Simoni M \& Huhtaniemi I (eds) Endocrinology of the Testis and Male Reproduction (Springer International Publishing, 2017).

54. Whitcomb RW \& Crowley WF Hypogonadotropic hypogonadism: gonadotropin-releasing hormone therapy. Curr. Ther. Endocrinol. Metab 6, 353-355 (1997). [PubMed: 9174769]

55. Spratt DI et al. The spectrum of abnormal patterns of gonadotropin-releasing hormone secretion in men with idiopathic hypogonadotropic hypogonadism: clinical and laboratory correlations. J. Clin. Endocrinol. Metab 64, 283-291 (1987). [PubMed: 3098771]

56. Raivio T et al. Reversal of idiopathic hypogonadotropic hypogonadism. N. Engl. J. Med 357, 863873 (2007). [PubMed: 17761590]

57. Sidhoum VF et al. Reversal and relapse of hypogonadotropic hypogonadism: resilience and fragility of the reproductive neuroendocrine system. J. Clin. Endocrinol. Metab 99, 861-870 (2014). [PubMed: 24423288] This study is of paramount relevance in the discussion of the potential reversal of idiopathic hypogonadotropic hypogonadism.

58. Pierzchlewska MM, Robaczyk MG \& Vogel I Induction of puberty with human chorionic gonadotropin (hCG) followed by reversal of hypogonadotropic hypogonadism in Kallmann syndrome. Endokrynol. Pol 68, 692-696 (2015).

59. Santhakumar A, Balasubramanian R, Miller M \& Quinton R Reversal of isolated hypogonadotropic hypogonadism: long-term integrity of hypothalamo-pituitary-testicular axis in two men is dependent on intermittent androgen exposure. Clin. Endocrinol 81, 473-476 (2013).

60. Finkelstein JS et al. Pulsatile gonadotropin secretion after discontinuation of long term gonadotropin-releasing hormone $(\mathrm{GnRH})$ administration in a subset of GnRH-deficient men. J. Clin. Endocrinol. Metab 69, 377-385 (1989). [PubMed: 2502554]

61. Pitteloud $\mathrm{N}$ et al. The fertile eunuch variant of idiopathic hypogonadotropic hypogonadism: spontaneous reversal associated with a homozygous mutation in the gonadotropin-releasing hormone receptor 1. J. Clin. Endocrinol. Metab 86, 2470-2475 (2001). [PubMed: 11397842]

62. Pitteloud N et al. Reversible Kallmann syndrome, delayed puberty, and isolated anosmia occurring in a single family with a mutation in the fibroblast growth factor receptor 1 gene. J. Clin. Endocrinol. Metab 90, 1317-1322 (2005). [PubMed: 15613419]

63. Laitinen E-M et al. Reversible congenital hypogonadotropic hypogonadism in patients with CHD7, FGFR1 or GNRHR mutations. PLOS ONE 7, e39450 (2012). [PubMed: 22724017]

64. Waldstreicher $\mathrm{J}$ et al. The genetic and clinical heterogeneity of gonadotropin-releasing hormone deficiency in the human. J. Clin. Endocrinol. Metab 81, 4388-4395 (1996). [PubMed: 8954047]

65. Dodé $\mathrm{C}$ et al. Loss-of-function mutations in FGFR1 cause autosomal dominant Kallmann syndrome. Nat. Genet 33, 463-465 (2003). [PubMed: 12627230] 
66. Ayari B \& Soussi-Yanicostas N FGFR1 and anosmin-1 underlying genetically distinct forms of Kallmann syndrome are co-expressed and interact in olfactory bulbs. Dev. Genes Evol 217, 169_ 175 (2007). [PubMed: 17186267]

67. Topaloglu AK et al. Inactivating KISS1 mutation and hypogonadotropic hypogonadism. N. Engl. J. Med 366, 629-635 (2012). [PubMed: 22335740]

68. Silveira LG, Latronico AC \& Seminara SB Kisspeptin and clinical disorders. Adv. Exp. Med. Biol 784, 187-199 (2013). [PubMed: 23550007]

69. Goodman RL et al. Kisspeptin neurons in the arcuate nucleus of the ewe express both dynorphin A and neurokinin B. Endocrinology 148, 5752-5760 (2007). [PubMed: 17823266]

70. Ramaswamy S et al. Neurokinin B stimulates GnRH release in the male monkey (Macaca mulatta) and is colocalized with kisspeptin in the arcuate nucleus. Endocrinology 151, 4494-4503 (2010). [PubMed: 20573725]

71. Gianetti E et al. TAC3/TACR3 mutations reveal preferential activation of gonadotropin-releasing hormone release by neurokinin B in neonatal life followed by reversal in adulthood. J. Clin. Endocrinol. Metab 95, 2857-2867 (2010). [PubMed: 20332248]

72. Zhu J et al. A shared genetic basis for self-limited delayed puberty and idiopathic hypogonadotropic hypogonadism. J. Clin. Endocrinol. Metab 100, E646-E654 (2015). [PubMed: 25636053]

73. Perry JR et al. Parent-of-origin-specific allelic associations among 106 genomic loci for age at menarche. Nature 514, 92-97 (2014). [PubMed: 25231870]

74. Day FR et al. Genomic analyses identify hundreds of variants associated with age at menarche and support a role for puberty timing in cancer risk. Nat. Genet 49, 834-841 (2017). [PubMed: 28436984]

75. Shi Z, Araujo AB, Martin S, O’Loughlin P \& Wittert GA Longitudinal changes in testosterone over five years in community-dwelling men. J. Clin. Endocrinol. Metab 98, 3289-3297 (2013). [PubMed: 23775354]

76. Wu FCW et al. Hypothalamic-pituitary-testicular axis disruptions in older men are differentially linked to age and modifiable risk factors: the European Male Aging Study. J. Clin. Endocrinol. Metab 93, 2737-2745 (2008). [PubMed: 18270261]

77. Feldman HA et al. Age trends in the level of serum testosterone and other hormones in middleaged men: longitudinal results from the Massachusetts Male Aging Study. J. Clin. Endocrinol. Metab 87, 589-598 (2002). [PubMed: 11836290]

78. Rastrelli G et al. Symptomatic androgen deficiency develops only when both total and free testosterone decline in obese men who may have incident biochemical secondary hypogonadism: Prospective results from the EMAS. Clin. Endocrinol 89, 459-469 (2018).

79. Perheentupa A \& Huhtaniemi I Aging of the human ovary and testis. Mol. Cell. Endocrinol 299, 213 (2009). [PubMed: 19059459]

80. Camacho EM et al. Age-associated changes in hypothalamic-pituitary-testicular function in middle-aged and older men are modified by weight change and lifestyle factors: longitudinal results from the European Male Ageing Study. Eur. J. Endocrinol 168, 445-455 (2013). [PubMed: 23425925]

81. Corona $\mathrm{G}$ et al. Body weight loss reverts obesity-associated hypogonadotropic hypogonadism: a systematic review and meta-analysis. Eur. J. Endocrinol 168, 829-843 (2013). [PubMed: 23482592] This study reports the findings of a systematic review and meta-analysis that outline that weight gain suppresses and weight loss increases circulating testosterone levels.

82. Corona G, Vignozzi L, Sforza A, Mannucci E \& Maggi M Obesity and late-onset hypogonadism. Mol. Cell. Endocrinol 418, 120-133 (2015). [PubMed: 26143633]

83. Antonio L et al. Low free testosterone is associated with hypogonadal signs and symptoms in men with normal total testosterone. J. Clin. Endocrinol. Metab 101, 2647-2657 (2016). [PubMed: 26909800]

84. Tsatsanis $\mathrm{C}$ et al. The impact of adipose tissue-derived factors on the hypothalamic-pituitarygonadal (HPG) axis. Horm. Athens Greece 14, 549-562 (2015).

85. Brüning JC et al. Role of brain insulin receptor in control of body weight and reproduction. Science 289, 2122-2125 (2000). [PubMed: 11000114] 
86. Tena-Sempere M Interaction between energy homeostasis and reproduction: central effects of leptin and ghrelin on the reproductive axis. Horm. Metab. Res 45, 919-927 (2013). [PubMed: 24155252]

87. Luukkaa V et al. Inverse correlation between serum testosterone and leptin in men. J. Clin. Endocrinol. Metab 83, 3243-3246 (1998). [PubMed: 9745436]

88. Isidori AM et al. Leptin and androgens in male obesity: evidence for leptin contribution to reduced androgen levels. J. Clin. Endocrinol. Metab 84, 3673-3680 (1999). [PubMed: 10523013]

89. Landry D, Cloutier F \& Martin LJ Implications of leptin in neuroendocrine regulation of male reproduction. Reprod. Biol 13, 1-14 (2013). [PubMed: 23522066]

90. Banks WA et al. Triglycerides induce leptin resistance at the blood-brain barrier. Diabetes 53, 1253-1260 (2004). [PubMed: 15111494]

91. Ye Z, Liu G, Guo J \& Su Z Hypothalamic endoplasmic reticulum stress as a key mediator of obesity-induced leptin resistance. Obes. Rev 19, 770-785 (2018). [PubMed: 29514392]

92. Pittas AG, Joseph NA \& Greenberg AS Adipocytokines and insulin resistance. J. Clin. Endocrinol. Metab 89, 447-452 (2004). [PubMed: 14764746]

93. Veldhuis J, Vkng R, Roelfsema F \& Takahashi P Proinflammatory cytokine infusion attenuates LH Itextquotesingles feedforward on testosterone secretion: modulation by age. J. Clin. Endocrinol. Metab 101, 539-549 (2016). [PubMed: 26600270]

94. Pagotto U, Marsicano G, Cota D, Lutz B \& Pasquali R The emerging role of the endocannabinoid system in endocrine regulation and energy balance. Endocr. Rev 27, 73-100 (2006). [PubMed: 16306385]

95. Porte D, Baskin DG \& Schwartz MW Insulin signaling in the central nervous system: a critical role in metabolic homeostasis and disease from C. elegans to humans. Diabetes 54, 1264-1276 (2005). [PubMed: 15855309]

96. George JT, Millar RP \& Anderson RA Hypothesis: kisspeptin mediates male hypogonadism in obesity and type 2 diabetes. Neuroendocrinology 91, 302-307 (2010). [PubMed: 20628262]

97. Aarts E et al. Gonadal status and outcome of bariatric surgery in obese men. Clin. Endocrinol 81, 378-386 (2013).

98. Pellitero S et al. Hypogonadotropic hypogonadism in morbidly obese males is reversed after bariatric surgery. Obes. Surg 22, 1835-1842 (2012). [PubMed: 22923309]

99. Chosich $\mathrm{J}$ et al. Acute recapitulation of the hyperinsulinemia and hyperlipidemia characteristic of metabolic syndrome suppresses gonadotropins. Obesity 25, 553-560 (2017). [PubMed: 28158916]

100. Grinspon RP et al. Gonadotrophin secretion pattern in anorchid boys from birth to pubertal age: pathophysiological aspects and diagnostic usefulness. Clin. Endocrinol 76, 698-705 (2012).

101. Grinspon RP \& Rey RA Anti-müllerian hormone and sertoli cell function in paediatric male hypogonadism. Horm. Res. Paediatr 73, 81-92 (2010). [PubMed: 20190544]

102. Grinspon RP et al. Early onset of primary hypogonadism revealed by serum anti-Müllerian hormone determination during infancy and childhood in trisomy 21. Int. J. Androl 34, e487-e498 (2011). [PubMed: 21831236]

103. Bastida MG et al. Establishment of testicular endocrine function impairment during childhood and puberty in boys with Klinefelter syndrome. Clin. Endocrinol 67, 863-870 (2007).

104. Boehm U et al. Expert consensus document: European Consensus Statement on congenital hypogonadotropic hypogonadism - pathogenesis, diagnosis and treatment. Nat. Rev. Endocrinol 11, 547-564 (2015). [PubMed: 26194704] This manuscript reports the findings of an expert consensus regarding the pathogenesis, diagnosis and treatment of congenital hypogonadotropic hypogonadism.

105. Palmert MR \& Dunkel L Clinical practice. Delayed puberty. N. Engl. J. Med 366, 443-453 (2012). [PubMed: 22296078]

106. Grinspon RP et al. Basal follicle-stimulating hormone and peak gonadotropin levels after gonadotropin-releasing hormone infusion show high diagnostic accuracy in boys with suspicion of hypogonadotropic hypogonadism. J. Clin. Endocrinol. Metab 95, 2811-2818 (2010). [PubMed: 20371659] 
107. Coutant R et al. Baseline inhibin B and anti-Mullerian hormone measurements for diagnosis of hypogonadotropic hypogonadism $(\mathrm{HH})$ in boys with delayed puberty. J. Clin. Endocrinol. Metab 95, 5225-5232 (2010). [PubMed: 20826577]

108. Sykiotis GP et al. Congenital idiopathic hypogonadotropic hypogonadism: evidence of defects in the hypothalamus, pituitary, and testes. J. Clin. Endocrinol. Metab 95, 3019-3027 (2010). [PubMed: 20382682]

109. Au MG, Crowley WF \& Buck CL Genetic counseling for isolated GnRH deficiency. Mol. Cell. Endocrinol 346, 102-109 (2011). [PubMed: 21664415]

110. Stamou MI, Cox KH \& Crowley WF Discovering genes essential to the hypothalamic regulation of human reproduction using a human disease model: adjusting to life in the "-omics" era. Endocr. Rev 36, 603-621 (2015). [PubMed: 26394276]

111. Bhasin $\mathrm{S}$ et al. Testosterone therapy in men with androgen deficiency syndromes: an Endocrine Society Clinical Practice Guideline. J. Clin. Endocrinol. Metab 95, 2536-2559 (2010). [PubMed: 20525905]

112. Bhasin S et al. Testosterone therapy in men with hypogonadism: an Endocrine Society Clinical Practice Guideline. J. Clin. Endocrinol. Metab 103, 1715-1744 (2018). [PubMed: 29562364] This manuscript details the most updated clinical practice guidelines recently released by the Endocrine Society.

113. Hackett $\mathrm{G}$ et al. British Society for Sexual Medicine Guidelines on adult testosterone deficiency, with statements for UK practice. J. Sex. Med 14, 1504-1523 (2017). [PubMed: 29198507] This manuscript reports the most recent recommendations released by the BSSM on adult testosterone deficiency, even as a part of the real-life experience.

114. Petak SM et al. American Association of Clinical Endocrinologists Medical Guidelines for clinical practice for the evaluation and treatment of hypogonadism in adult male patients-2002 update. Endocr. Pract 8, 440-456 (2002). [PubMed: 15260010] This paper is a pivotal manuscript in the field of diagnostic work-up and treatment of adult male patients with hypogonadism.

115. Jasuja GK, Bhasin S, Reisman JI, Berlowitz DR \& Rose AJ Ascertainment of testosterone prescribing practices in the VA Med. Care 53, 746-752 (2015). [PubMed: 26196850]

116. Baillargeon J, Kuo Y-F, Westra JR, Urban RJ \& Goodwin JS Testosterone prescribing in the United States, 2002-2016. JAMA 320, 200 (2018). [PubMed: 29998328]

117. Snyder PJ et al. Effects of testosterone treatment in older men. N. Engl. J. Med 374, 611-624 (2016). [PubMed: 26886521]

118. Snyder PJ et al. Effect of testosterone treatment on bone mineral density in men over 65 years of age. J. Clin. Endocrinol. Metab 84, 1966-1972 (1999). [PubMed: 10372695]

119. Walther A, Breidenstein J \& Miller R Association of testosterone treatment with alleviation of depressive symptoms in men: a systematic review and meta-analysis. JAMA Psychiatry 76, 31 (2019). [PubMed: 30427999]

120. European Association of Urology. EAU Guidelines on male hypogonadism. EAU https:// uroweb.org/guideline/male-hypogonadism/ (2018).

121. Taylor AE, Keevil B \& Huhtaniemi IT Mass spectrometry and immunoassay: how to measure steroid hormones today and tomorrow. Eur. J. Endocrinol 173, D1-D12 (2015). [PubMed: 25877990]

122. Basaria $\mathrm{S}$ et al. Effects of testosterone administration for 3 years on subclinical atherosclerosis progression in older men with low or low-normal testosterone levels. JAMA 314, 570 (2015). [PubMed: 26262795]

123. Glass AR, Swerdloff RS, Bray GA, Dahms WT \& Atkinson RL Low serum testosterone and sexhormone-binding-globulin in massively obese men. J. Clin. Endocrinol. Metab 45, 1211-1219 (1977). [PubMed: 338622]

124. Stanworth RD \& Jones TH Testosterone for the aging male; current evidence and recommended practice. Clin. Interv. Aging 3, 25-44 (2008). [PubMed: 18488876]

125. Vermeulen A, Verdonck L \& Kaufman JM A critical evaluation of simple methods for the estimation of free testosterone in serum. J. Clin. Endocrinol. Metab 84, 3666-3672 (1999). [PubMed: 10523012] This manuscript describes the most widely used formula for determining the calculated free testosterone value. 
126. Giton F Serum bioavailable testosterone: assayed or calculated? Clin. Chem 52, 474-481 (2006). [PubMed: 16384884]

127. Hackbarth JS, Hoyne JB, Grebe SK \& Singh RJ Accuracy of calculated free testosterone differs between equations and depends on gender and SHBG concentration. Steroids 76, 48-55 (2011). [PubMed: 20816687]

128. Boeri L et al. Does calculated free testosterone overcome total testosterone in protecting from sexual symptom impairment? Findings of a cross-sectional study. J. Sex. Med 14, 1549-1557 (2017). [PubMed: 29198510]

129. Andrade-Rocha FT Semen analysis in laboratory practice: an overview of routine tests. J. Clin. Lab. Anal 17, 247-258 (2003). [PubMed: 14614749]

130. Rhoden EL et al. The value of pituitary magnetic resonance imaging in men with hypogonadism. J. Urol 170, 795-798 (2003). [PubMed: 12913700]

131. Molitch ME Diagnosis and treatment of pituitary adenomas. JAMA 317, 516 (2017). [PubMed: 28170483]

132. Chiloiro $\mathrm{S}$ et al. Diagnosis of endocrine disease: primary empty sella: a comprehensive review. Eur J. Endocrinol 177, R275-R285 (2017). [PubMed: 28780516]

133. Corona G, Rastrelli G, Vignozzi L \& Maggi M Emerging medication for the treatment of male hypogonadism. Expert Opin. Emerg. Drugs 17, 239-259 (2012). [PubMed: 22612692]

134. Corona G, Ratrelli G \& Maggi M The pharmacotherapy of male hypogonadism besides androgens. Expert Opin. Pharmacother 16, 369-387 (2014). [PubMed: 25523084]

135. Rey RA \& Grinspon RP Normal male sexual differentiation and aetiology of disorders of sex development. Best Pract. Res. Clin. Endocrinol. Metab 25, 221-238 (2011). [PubMed: 21397195]

136. Lee PA et al. Global Disorders of Sex Development Update since 2006: perceptions, approach and care. Horm. Res. Paediatr 85, 158-180 (2016). [PubMed: 26820577]

137. Howard S \& Dunkel L Sex steroid and gonadotropin treatment in male delayed puberty. Endocr. Dev 29, 185-197 (2016). [PubMed: 26680579]

138. Wei C \& Crowne EC Recent advances in the understanding and management of delayed puberty. Arch. Dis. Child 101, 481-488 (2015). [PubMed: 26353794] This manuscript summarizes recent advances regarding the neuroendocrine, genetic and environmental factors controlling pubertal development, with potential correlations in terms of delayed puberty pathophysiology.

139. Giri D et al. Testosterone therapy improves the first year height velocity in adolescent boys with constitutional delay of growth and puberty. Int. J. Endocrinol. Metab 15, e42311 (2017). [PubMed: 28848608]

140. Richman RA \& Kirsch LR Testosterone treatment in adolescent boys with constitutional delay in growth and development. N. Engl. J. Med 319, 1563-1567 (1988). [PubMed: 3200264]

141. Spratt DI et al. Subcutaneous injection of testosterone is an effective and preferred alternative to intramuscular injection: demonstration in female-to-male transgender patients. J. Clin. Endocrinol. Metab 102, 2349-2355 (2017). [PubMed: 28379417]

142. Chioma L, Papucci C, Fintini D \& Cappa M Use of testosterone gel compared to intramuscular formulation for puberty induction in males with constitutional delay of growth and puberty: a preliminary study. J. Endocrinol. Invest 41, 259-263 (2017). [PubMed: 28695484]

143. Rogol AD et al. A multicenter, open-label, observational study of testosterone gel $(1 \%)$ in the treatment of adolescent boys with Klinefelter syndrome or anorchia. J. Adolesc. Health 54, 20 25 (2014). [PubMed: 24035132]

144. Rogol AD, Tkachenko N \& Bryson N Natesto ${ }^{\mathrm{TM}}$, a novel testosterone nasal gel, normalizes androgen levels in hypogonadal men. Andrology 4, 46-54 (2015). [PubMed: 26695758]

145. Decourt $C$ et al. A synthetic kisspeptin analog that triggers ovulation and advances puberty. Sci. Rep 6, 26908 (2016). [PubMed: 27245315]

146. Shulman DI, Francis GL, Palmert MR \& Eugster E A Use of aromatase inhibitors in children and adolescents with disorders of growth and adolescent development. Pediatrics 121, e975-e983 (2008). [PubMed: 18381525]

147. Wit JM, Hero M \& Nunez SB Aromatase inhibitors in pediatrics. Nat. Rev. Endocrinol 8, 135147 (2011). [PubMed: 22024975] 
148. Varimo $\mathrm{T}$ et al. Letrozole versus testosterone for promotion of endogenous puberty in boys with constitutional delay of growth and puberty: a randomised controlled phase 3 trial. Lancet Child Adolesc. Health 3, 109-120 (2019). [PubMed: 30612946]

149. Rohayem J, Hauffa BP, Zacharin M, Kliesch S \& Zitzmann M Testicular growth and spermatogenesis: new goals for pubertal hormone replacement in boys with hypogonadotropic hypogonadism? -a multicentre prospective study of hCG/rFSH treatment outcomes during adolescence-. Clin. Endocrinol 86, 75-87 (2016).

150. Gong C, Liu Y, Qin M, Wu D \& Wang X Pulsatile GnRH is superior to hCG in therapeutic efficacy in adolescent boys with hypogonadotropic hypogonadodism. J. Clin. Endocrinol. Metab 100, 2793-2799 (2015). [PubMed: 25978110]

151. Rastrelli G, Corona G, Mannucci E \& Maggi M Factors affecting spermatogenesis upon gonadotropin-replacement therapy: a meta-analytic study. Andrology 2, 794-808 (2014). [PubMed: 25271205]

152. Aksglaede L \& Juul A Therapy of endocrine disease: testicular function and fertility in men with Klinefelter syndrome: a review. Eur. J. Endocrinol 168, R67-R76 (2013). [PubMed: 23504510]

153. Forti G, Corona G, Vignozzi L, Krausz C \& Maggi M Klinefelter's syndrome: a clinical and therapeutical update. Sex. Dev 4, 249-258 (2010). [PubMed: 20664188]

154. Corona $\mathrm{G}$ et al. Sexual dysfunction in subjects with Klinefelter's syndrome. Int. J. Androl 33, 574-580 (2009). [PubMed: 19780865]

155. Vignozzi L, Corona G, Forti G, Jannini EA \& Maggi M Clinical and therapeutic aspects of Klinefelter's syndrome: sexual function. Mol. Hum. Reprod 16, 418-424 (2010). [PubMed: 20348547]

156. Corona G et al. Sperm recovery and ICSI outcomes in Klinefelter syndrome: a systematic review and meta-analysis. Hum. Reprod. Update 23, 265-275 (2017). [PubMed: 28379559] This manuscript reports novel findings of a meta-analysis devoted to defining positive sperm retrieval outcomes at surgery in men with Klinefelter syndrome.

157. Nahata L et al. Sperm retrieval in adolescents and young adults with Klinefelter syndrome: a prospective, pilot study. J. Pediatr 170, 260-265 (2016). [PubMed: 26746120]

158. Huhtaniemi1. Late-onset hypogonadism: current concepts and controversies of pathogenesis, diagnosis and treatment. Asian J. Androl 16, 192 (2014). [PubMed: 24407185]

159. Rastrelli $\mathrm{G}$ et al. Development of and recovery from secondary hypogonadism in aging men: prospective results from the EMAS. J. Clin. Endocrinol. Metab 100, 3172-3182 (2015). [PubMed: 26000545] This manuscripts reports the findings of the EMAS study, a prospective observational general population cohort survey, which outlines that obesity-related metabolic and lifestyle factors predispose older men to the development of secondary hypogonadism, which is frequently reversible with weight loss.

160. Kumagai $\mathrm{H}$ et al. Vigorous physical activity is associated with regular aerobic exercise-induced increased serum testosterone levels in overweight/obese men. Horm. Metab. Res 50, 73-79 (2018). [PubMed: 28934816]

161. Shao $\mathrm{N}$ et al. Short-term combined treatment with exenatide and metformin is superior to glimepiride combined metformin in improvement of serum testosterone levels in type 2 diabetic patients with obesity. Andrologia 50, e13039 (2018). [PubMed: 29740874]

162. Ng Tang Fui M, Hoermann R, Zajac JD \& Grossmann M The effects of testosterone on body composition in obese men are not sustained after cessation of testosterone treatment. Clin. Endocrinol 87, 336-343 (2017).

163. Brock $\mathrm{G}$ et al. Effect of testosterone solution $2 \%$ on testosterone concentration, sex drive and energy in hypogonadal men: results of a placebo controlled study. J. Urol 195, 699-705 (2016). [PubMed: 26498057]

164. Hackett $\mathrm{G}$ et al. Testosterone undecanoate improves sexual function in men with type 2 diabetes and severe hypogonadism: results from a 30-week randomized placebo-controlled study. BJU Int. 118, 804-813 (2016). [PubMed: 27124889]

165. Hackett $\mathrm{G}$ et al. Testosterone replacement therapy improves metabolic parameters in hypogonadal men with type 2 diabetes but not in men with coexisting depression: the BLAST study. J. Sex. Med 11, 840-856 (2014). [PubMed: 24308723] 
166. Jones TH et al. Testosterone replacement in hypogonadal men with type 2 diabetes and/or metabolic syndrome (the T1MES2 study). Diabetes Care 34, 828-837 (2011). [PubMed: 21386088]

167. Tracz MJ et al. Testosterone use in men and its effects on bone health. A systematic review and meta-analysis of randomized placebo-controlled trials. J. Clin. Endocrinol. Metab 91, 2011-2016 (2006). [PubMed: 16720668]

168. Kenny AM et al. Dehydroepiandrosterone combined with exercise improves muscle strength and physical function in frail older women. J. Am. Geriatr. Soc 58, 1707-1714 (2010). [PubMed: 20863330]

169. Snyder PJ et al. Effect of testosterone treatment on volumetric bone density and strength in older men with low testosterone. JAMA Intern. Med 177, 471 (2017). [PubMed: 28241231]

170. Gennari L \& Bilezikian JP New and developing pharmacotherapy for osteoporosis in men. Expert Opin. Pharmacother 19, 253-264 (2018). [PubMed: 29350069]

171. Rochira V, Antonio L \& Vanderschueren D EAA clinical guideline on management of bone health in the andrological outpatient clinic. Andrology 6, 272-285 (2018). [PubMed: 29499097]

172. Bachman E et al. Testosterone induces erythrocytosis via increased erythropoietin and suppressed hepcidin: evidence for a new erythropoietin/hemoglobin set point. J. Gerontol. A 69, 725-735 (2014).

173. Roy CN et al. Association of testosterone levels with anemia in older men: a controlled clinical trial. JAMA Intern. Med 177, 480 (2017). [PubMed: 28241237]

174. Khera $\mathrm{M}$ et al. The effect of testosterone supplementation on depression symptoms in hypogonadal men from the Testim Registry in the US (TRiUS). Aging Male 15, 14-21 (2011). [PubMed: 22092151]

175. Schneider G et al. Depressive symptoms in men aged 50 years and older and their relationship to genetic androgen receptor polymorphism and sex hormone levels in three different samples. Am. J. Geriatr. Psychiatry 19, 274-283 (2011). [PubMed: 20808127]

176. Schneider G, Zitzmann M, Gromoll J, Ladwig KH \& Berger K The relation between sex hormone levels, the androgen receptor CAGn-polymorphism and depression and mortality in older men in a community study. Psychoneuroendocrinology 38, 2083-2090 (2013). [PubMed: 23602133]

177. Resnick SM et al. Testosterone treatment and cognitive function in older men with low testosterone and age-associated memory impairment. JAMA 317, 717-727 (2017). [PubMed: 28241356]

178. Bhasin S et al. Effect of testosterone replacement on measures of mobility in older men with mobility limitation and low testosterone concentrations: secondary analyses of the Testosterone Trials. Lancet Diabetes Endocrinol. 6, 879-890 (2018). [PubMed: 30366567]

179. Holmegard HN, Nordestgaard BG, Schnohr P, Tybjaerg-Hansen A \& Benn M Endogenous sex hormones and risk of venous thromboembolism in women and men. J. Thromb. Haemost 12, 297-305 (2014). [PubMed: 24329981]

180. Baillargeon J, Kuo Y-F, Fang X \& Shahinian VB Long-term exposure to testosterone therapy and the risk of high grade prostate cancer. J. Urol 194, 1612-1616 (2015). [PubMed: 26066403]

181. Dohle GR, Smit M \& Weber RFA Androgens and male fertility. World J. Urol 21, 341-345 (2003). [PubMed: 14566423]

182. Soisson V et al. A J-shaped association between plasma testosterone and risk of ischemic arterial event in elderly men: the French 3C cohort study. Maturitas 75, 282-288 (2013). [PubMed: 23706278]

183. Corona $\mathrm{G}$ et al. Endogenous testosterone levels and cardiovascular risk: meta-analysis of observational studies. J. Sex. Med 15, 1260-1271 (2018). [PubMed: 30145097]

184. Saad F, Röhrig G, von Haehling S \& Traish A Testosterone deficiency and testosterone treatment in older men. Gerontology 63, 144-156 (2016). [PubMed: 27855417]

185. Corona $\mathrm{G}$ et al. Testosterone supplementation and body composition: results from a meta-analysis of observational studies. J. Endocrinol. Invest 39, 967-981 (2016). [PubMed: 27241317]

186. Corona $\mathrm{G}$ et al. Therapy of endocrine disease: testosterone supplementation and body composition: results from a meta-analysis study. Eur. J. Endocrinol 174, R99-R116 (2016). [PubMed: 26537862] 
187. Corona G, Rastrelli G, Reisman Y, Sforza A \& Maggi M The safety of available treatments of male hypogonadism in organic and functional hypogonadism. Expert Opin. Drug Saf 17, 277 292 (2018). [PubMed: 29334271]

188. Etminan M, Skeldon SC, Goldenberg SL, Carleton B \& Brophy JM Testosterone therapy and risk of myocardial infarction: a pharmacoepidemiologic study. Pharmacotherapy 35, 72-78 (2015). [PubMed: 25582846]

189. US Food \& Drug Administration. FDA Drug Safety Communication: FDA cautions about using testosterone products for low testosterone due to aging; requires labeling change to inform of possible increased risk of heart attack and stroke with use. FDA.gov https://www.fda.gov/Drugs/ DrugSafety/ucm436259.htm (updated 26 Feb 2018).

190. Government of Canada Drug and Health Product Register. Summary safety review — testosterone replacement products - Health Canada. Health Canada https://hpr-rps.hres.ca/reg-content/ summary-safety-review-detail.php?linkID=SSR00058 (2014).

191. Yeap BB et al. Endocrine Society of Australia position statement on male hypogonadism (part 1): assessment and indications for testosterone therapy. Med. J. Aust 205, 173-178 (2016). [PubMed: 27510348]

192. European Medicines Agency. Testosterone-containing medicines Europa.eu https:// www.ema.europa.eu/en/medicines/human/referrals/testosterone-containing-medicines (updated 8 Jan 2015).

193. Corona $\mathrm{G}$ et al. Testosterone and cardiovascular risk: meta-analysis of interventional studies. J. Sex. Med 15, 820-838 (2018). [PubMed: 29803351]

194. Luo S, Au Yeung SL, Zhao JV, Burgess S \& Schooling CM Association of genetically predicted testosterone with thromboembolism, heart failure, and myocardial infarction: mendelian randomisation study in UK Biobank. BMJ 364, 1476 (2019).

195. Coviello AD et al. Effects of graded doses of testosterone on erythropoiesis in healthy young and older men. J. Clin. Endocrinol. Metab 93, 914-919 (2008). [PubMed: 18160461]

196. Hoyos CM, Killick R, Yee BJ, Grunstein RR \& Liu PY Effects of testosterone therapy on sleep and breathing in obese men with severe obstructive sleep apnoea: a randomized placebocontrolled trial. Clin. Endocrinol 77, 599-607 (2012).

197. Corona $\mathrm{G}$ et al. Meta-analysis of results of testosterone therapy on sexual function based on international index of erectile function scores. Eur. Urol 72, 1000-1011 (2017). [PubMed: 28434676] This manuscript is clinically relevant in saying that testosterone therapy significantly improves erectile function in men with more severe hypogonadism compared with those with milder testosterone deficiency.

198. Ponce OJ et al. The efficacy and adverse events of testosterone replacement therapy in hypogonadal men: a systematic review and meta-analysis of randomized, placebo-controlled trials. J. Clin. Endocrinol. Metab 103, 1745-1754 (2018).

199. Kaufman JM, Lapauw B, Mahmoud A, T’Sjoen G \& Huhtaniemi IT Aging and the male reproductive system. Endocr. Rev https://d0i.Org/10.1210/er.2018-00178 (2019).

200. Isidori AM et al. Effects of testosterone on sexual function in men: results of a meta-analysis. Clin. Endocrinol 63, 381-394 (2005).

201. Hatzimouratidis K et al. Pharmacotherapy for erectile dysfunction: recommendations from the Fourth International Consultation for Sexual Medicine (ICSM 2015). J. Sex. Med 13, 465-488 (2016). [PubMed: 27045254]

202. Isidori AM et al. A critical analysis of the role of testosterone in erectile function: from pathophysiology to treatment-a systematic review. Eur. Urol 65, 99-112 (2014). [PubMed: 24050791] This manuscript describes the findings of a critical reappraisal of the role of testosterone in terms of erectile function physiology and pathophysiology.

203. Corona $\mathrm{G}$ et al. Testosterone supplementation and sexual function: a meta-analysis study. J. Sex. Med 11, 1577-1592 (2014). [PubMed: 24697970]

204. Belling $\mathrm{K}$ et al. Klinefelter syndrome comorbidities linked to increased X chromosome gene dosage and altered protein interactome activity. Hum. Mol. Genet 26, 1219-1229 (2017). [PubMed: 28369266] 
205. D'Aurora M et al. Testis transcriptome modulation in Klinefelter patients with hypospermatogenesis. Sci. Rep 7, 45729 (2017). [PubMed: 28361989]

206. Winge SB et al. Transcriptome profiling of fetal Klinefelter testis tissue reveals a possible involvement of long non-coding RNAs in gonocyte maturation. Hum. Mol. Genet 27, 430-439 (2017).

207. Cimino L et al. Decreased miRN A expression in Klinefelter syndrome. Sci. Rep 7, 16672 (2017). [PubMed: 29192217]

208. Wan ES et al. Genome-wide site-specific differential methylation in the blood of individuals with Klinefelter syndrome. Mol. Reprod. Dev 82, 377-386 (2015). [PubMed: 25988574]

209. Samango-Sprouse $C$ et al. Positive effects of early androgen therapy on the behavioral phenotype of boys with 47,XXY. Am. J. Med. Genet. C 169, 150-157 (2015).

210. Harrington J \& Palmert MR Distinguishing constitutional delay of growth and puberty from isolated hypogonadotropic hypogonadism: critical appraisal of available diagnostic tests. J. Clin. Endocrinol. Metab 97, 3056-3067 (2012). [PubMed: 22723321] This manuscript critically deals with the difficulties in distinguishing CDGP from isolated hypogonadotropic hypogonadism.

211. Xu C et al. Genetic testing facilitates prepubertal diagnosis of congenital hypogonadotropic hypogonadism. Clin. Genet 92, 213-216 (2017). [PubMed: 28195315]

212. Chan Y-M, Lippincott MF, Kusa TO \& Seminara SB Divergent responses to kisspeptin in children with delayed puberty. JCI Insight 3, 99109 (2018). [PubMed: 29669934]

213. Stoupa A et al. Efficacy and safety of continuous subcutaneous infusion of recombinant human gonadotropins for congenital micropenis during early infancy. Horm. Res. Paediatr 87, 103-110 (2017). [PubMed: 28081535]

214. Main K, Schmidt I, Toppari J \& Skakkebaek N Early postnatal treatment of hypogonadotropic hypogonadism with recombinant human FSH and LH. Eur. J. Endocrinol 146, 75-79 (2002). [PubMed: 11751071]

215. Bouvattier $\mathrm{C}$ et al. Neonatal gonadotropin therapy in male congenital hypogonadotropic hypogonadism. Nat Rev. Endocrinol 8, 172-182 (2011). [PubMed: 22009162]

216. Ryden L et al. ESC Guidelines on diabetes, pre-diabetes, and cardiovascular diseases developed in collaboration with the EASD. Eur. Heart J 34, 3035-3087 (2013). [PubMed: 23996285]

217. Corona $\mathrm{G}$ et al. Cardiovascular risk associated with testosterone-boosting medications: a systematic review and meta-analysis. Expert Opin. Drug Saf 13, 1327-1351 (2014). [PubMed: 25139126]

218. Calof $\mathrm{OM}$ et al. Adverse events associated with testosterone replacement in middle-aged and older men: a meta-analysis of randomized, placebo-controlled trials. J. Gerontol. A 60, 14511457 (2005).

219. Haddad RM et al. Testosterone and cardiovascular risk in men: a systematic review and metaanalysis of randomized placebo-controlled trials. Mayo Clin. Proc 82, 29-39 (2007). [PubMed: 17285783]

220. Fernández-Balsells MM et al. Adverse effects of testosterone therapy in adult men: a systematic review and meta-analysis. J. Clin. Endocrinol. Metab 95, 2560-2575 (2010). [PubMed: 20525906]

221. Xu L, Freeman G, Cowling BJ \& Schooling CM Testosterone therapy and cardiovascular events among men: a systematic review and meta-analysis of placebo-controlled randomized trials. BMC Med 11, 108 (2013). [PubMed: 23597181]

222. Borst SE et al. Cardiovascular risks and elevation of serum DHT vary by route of testosterone administration: a systematic review and meta-analysis. BMC Med. 12, 211 (2014). [PubMed: 25428524]

223. Albert SG \& Morley JE Testosterone therapy, association with age, initiation and mode of therapy with cardiovascular events: a systematic review. Clin. Endocrinol 85, 436-443 (2016).

224. Alexander GC Iyer G, Lucas E, Lin D \& Singh S Cardiovascular risks of exogenous testosterone use among men: a systematic review and meta-analysis. Value Health 19, A43 (2016).

225. Corona G, Forti G \& Maggi M Why can patients with erectile dysfunction be considered lucky? The association with testosterone deficiency and metabolic syndrome. Aging Male 11, 193-199 (2008). [PubMed: 19172551] 
226. Thyen U, Lanz K, Holterhus P-M \& Hiort O Epidemiology and initial management of ambiguous genitalia at birth in Germany. Horm. Res. Paediatr 66, 195-203 (2006).

227. Klonisch T, Fowler PA \& Hombach-Klonisch S Molecular and genetic regulation of testis descent and external genitalia development. Dev. Biol 270, 1-18 (2004). [PubMed: 15136137]

228. Ivell R \& Anand-Ivell R Biological role and clinical significance of insulin-like peptide 3. Curr. Opin. Endocrinol. Diabetes Obes 18, 210-216 (2011). [PubMed: 21415739]

229. Lasala C, Carré-Eusèbe D, Picard J-Y \& Rey R Subcellular and molecular mechanisms regulating anti-Müllerian hormone gene expression in mammalian and nonmammalian species. DNA Cell Biol. 23, 572-585 (2004). [PubMed: 15383177]

230. Lamminmäki A et al. Testosterone measured in inlancy predicts subsequent sex-typed behavior in boys and in girls. Horm. Behav 61, 611-616 (2012). [PubMed: 22373494]

231. Goldman AL et al. A Reappraisal of testosterone's binding in circulation: physiological and clinical implications. Endocr. Rev 38, 302-324 (2017). [PubMed: 28673039]

232. Rastrelli G, Corona G, Cipriani S, Mannucci E \& Maggi M Sex hormone-binding globulin is associated with androgen deficiency features independently of total testosterone. Clin. Endocrinol 88, 556-564 (2018).

233. Kathrins M \& Niederberger C Diagnosis and treatment of infertility-related male hormonal dysfunction. Nat. Rev. Urol 13, 309-323 (2016). [PubMed: 27091665]

234. Mulhall $\mathrm{J}$ et al. Evaluation and management of testosterone deficiency: AUA Guideline. AUAnet.org https://www.auanet.org/Documents/Guidelines/PDF/Testosterone\%20Website $\% 20$ Final(0).pdf (2018).

235. Morales A et al. Diagnosis and management of testosterone deficiency syndrome in men: clinical practice guideline. Can. Med. Assoc. J 187, 1369-1377 (2015). [PubMed: 26504097]

236. Lunenfeld B et al. Recommendations on the diagnosis, treatment and monitoring of hypogonadism in men. Aging Male 18, 5-15 (2015). [PubMed: 25657080] 


\section{Box 1 |}

\section{Classification of male hypogonadism}

Historically, classification of male hypogonadism has been based on the anatomical location of the derangement that leads to testis failure (that is, primary or secondary hypogonadism). More recently, it has been proposed to classify hypogonadism according to the age of the appearance of the testicular failure and, therefore, to the phenotype ${ }^{133,134}$. Male phenotype can be severely altered if there is a testosterone deficiency during early fetal life, whereas an eunuchoid phenotype is often present when testosterone deficiency emerges during puberty ${ }^{133,134}$. When testosterone deficiency manifests during adulthood, the phenotype is relatively vague and mostly sexual dysfunction is considered to have some specificity ${ }^{45,225}$. In 2017, Grossmann and Matsumoto $^{31}$ suggested a new classification of male hypogonadism, distinguishing functional hypogonadism from its organic counterpart. Organic hypogonadism is characterized by any proven pathology affecting the hypothalamic-pituitary-gonadal axis and should be treated with the conventional medications (gonadotropins or testosterone) accordingly. Functional hypogonadism is based on the absence of any recognized organic alterations in the hypothalamic-pituitary-gonadal axis and should be treated, first by resolving or improving the associated comorbidities ${ }^{31}$. 


\section{Box 2 |}

\section{Types and causes of hypogonadism}

\section{Primary hypogonadism}

Primary hypogonadism (also known as hypergonadotropic hypogonadism) is caused by an inherent defect within the testes. This condition is biochemically characterized by low or absent testosterone levels and high gonadotropins levels. Spermatogenesis is usually severely impaired and not responsive to hormonal therapy.

\section{Congenital causes}

- Trisomies such as Klinefelter syndrome ${ }^{\mathrm{a}}$ and Down syndrome

- $\quad$ Y-Chromosome microdeletions ${ }^{\mathrm{a}}$

- Testicular dysgenesis syndrome or other conditions associated with cryptorchidism $^{\mathrm{a}}$

- $\quad$ Disorders of sex development ${ }^{\mathrm{a}}$

- Myotonic dystrophy (a genetic disorder associated with impaired muscle function $)^{\mathrm{a}}$

\section{Acquired causes}

- $\quad$ Mumps-related orchitis (or other types of severe infection of the testes) ${ }^{\mathrm{a}}$

- Irradiation or chemotherapy ${ }^{\mathrm{a}}$

- Trauma to the testes or castration ${ }^{\mathrm{a}}$

- Chronic illnesses (such as chronic kidney disease, chronic obstructive pulmonary disease or HIV infection) ${ }^{\mathrm{b}}$

- Ketoconazole (antifungal medication) intake ${ }^{\mathrm{b}}$

- Chronic alcoholism ${ }^{b}$

- $\quad$ Older age $\mathrm{a}^{\mathrm{a}, \mathrm{b}}$

\section{Secondary hypogonadism}

Secondary hypogonadism (also known as central hypogonadism or hypogonadotropic hypogonadism) is caused by a dysfunction in the hypothalamus and/or the pituitary gland. This condition is biochemically characterized by low or inappropriately normal gonadotropins levels along with low total testosterone levels. Spermatogenesis is impaired but is usually responsive to hormonal therapy.

\section{Congenital causes}

- Kallmann syndrome ${ }^{\mathrm{a}}$

- Idiopathic secondary hypogonadism ${ }^{\mathrm{a}}$

\section{Acquired causes}


- $\quad$ Pituitary dysfunction due to a tumour, surgery, trauma (for example, those causing stalk injury), infection (for example, tuberculosis) or infiltrative diseases (such as Langerhans cell histiocytosis) ${ }^{\mathrm{a}}$

- Hypothalamic dysfunction (due to, for example, tumours or intracranial masses (such as craniopharyngioma)) ${ }^{\mathrm{a}}$

- Hyperprolactinaemia ${ }^{\mathrm{a}}$

- Chronic conditions (such as type 2 diabetes mellitus, haemochromatosis, hepatic steatosis and cirrhosis or coronary artery disease) ${ }^{\mathrm{b}}$

- $\quad$ Drug use (glucocorticoids, opioids, androgens, progestins, oestrogens or gonadotropin-releasing hormone analogues) ${ }^{\mathrm{b}}$

- $\quad$ Obesity

- Malnutrition, wasting or anorexia nervosa ${ }^{b}$

- $\quad$ Excessive exercise ${ }^{b}$

- $\quad$ Older age (with associated comorbidities) $)^{\mathrm{a}, \mathrm{b}}$

${ }^{a}$ Organic origin. ${ }^{b}$ Functional origin. 


\section{Box 3 |}

\section{Paediatric hypogonadism}

When the hormonal deficiency develops during the first trimester of fetal life, the clinical manifestation is a disorder of sex development, with a variable degree of hypovi rilization of external genitalia. A wide range of rare genetic disorders can be implied that affect gonadal development or sex hormone synthesis or action. Overall, the incidence of those latter conditions is 1 in 5,000 live male births ${ }^{136,226}$. Congenital hypogonadotropic hypogonadism, either isolated or with deficiency of multiple pituitary hormones (that is, combined), is typically established after sex differentiation has occurred (that is, after the first trimester). It can present clinically at birth with signs such as micropenis, cryptorchidism and/or micro-orchidism or later as delayed puberty ${ }^{17}$. 


\section{Box 4 |}

\section{Ontogeny of the HPG axis during fetal development}

During the first trimester, the testes drive the virilization of internal and external genitalia (that is, the biological development of sex differences) through placental human chorionic gonadotropin (hCG)-stimulated androgen secretion by Leydig cells. Regression of Müllerian ducts, the anlagen of the uterus and Fallopian tubes occurs in response to anti-Müllerian hormone (AMH) secreted by Sertoli cells independently of pituitary gonadotropins ${ }^{4}$. Subsequently, luteinizing hormone (LH) and hCG maintain Leydig cell differentiation characterized by the secretion of androgens and insulin-like factor 3 (INSL3), whereas follicle-stimulating hormone (FSH) regulates Sertoli cells ${ }^{33}$. Androgens, in particular the testosterone metabolite dihydrotestosterone (DHT), are the main hormones responsible for the growth of the penis and the scrotum in the second half of intrauterine life, and, together with INSL3, they direct the testicular descent ${ }^{227,228}$. Sertoli cells proliferate, therefore, increasing testicular volume. Basal AMH secretion is independent of gonadotropins; however, FSH increases AMH output by stimulating Sertoli cell proliferation and upregulating AMH expression in Sertoli cells ${ }^{229}$. FSH also induces inhibin B secretion, which exerts negative feedback on FSH at the pituitary level. Testosterone elevation during fetal development has also been suggested to play a part in human neurobehavioural sexual differentiation; indeed, testosterone potentially has a relevant relationship to human neural structure and function in fetal and/or neonatal life ${ }^{4}$ as well as neurobehavioural sexual differentiation during early infancy 230 .

HPG, hypothalamic-pituitary-gonadal. 


\section{Box 5}

\section{Treatment of CDGP and persistent hypogonadism in adolescents}

\section{Testosterone $^{\mathrm{a}}$}

Adverse effects associated with testosterone use include erythrocytosis, weight gain, prostate hyperplasia and transaminitis; high doses can cause premature epiphyseal closure. Testosterone should be used with caution when bone age is $<10$ years. Anabolic steroids are not recommended for the induction of secondary sexual characteristics.

- Testosterone esters (that is, testosterone enanthate, testosterone cypionate and testosterone propionate): these formulations are usually administered as intramuscular injections, but subcutaneous use is an emerging option. Treatment can be associated with local adverse effects (such as pain, erythema, inflammatory reaction and sterile abscess); priapism can occur in patients with sickle cell disease.

- Testosterone undecanoate: data and experience of the use of this formulation in constitutional delay of growth and puberty (CDGP) are limited.

- Testosterone gel: data and experience of the use of this formulation in CDGP are limited. The gel can cause local irritation, and close skin contact with others should be avoided after applying to prevent transfer of medication.

- Testosterone nasal gel: data and experience of the use of this formulation in CDGP are lacking. Formulation requires multiple doses per day per nostril, but the lack of risk of secondary transference is an advantage.

\section{Pulsatile GnRH}

This treatment is usually reserved for instances of persistent hypogonadotropic hypogonadism due to hypothalamic defects. However, it is sometimes used for treatment of CDGP to induce testicular maturation and when fertility is desired. Pulsatile gonadotropin-releasing hormone $(\mathrm{GnRH})^{\mathrm{b}}$ is the most physiologic form of replacement therapy if the primary defect affects the hypothalamus. This formulation is administered via subcutaneous pump and requires extensive experience.

\section{hCG combined with recombinant FSH or purified hMG}

This treatment is usually reserved for instances of persistent hypogonadotropic hypogonadism due to hypothalamic or pituitary defects. However, it is sometimes used for treatment of CDGP to induce testicular maturation and when fertility is desired. The treatment includes subcutaneous or intramuscular human chorionic gonadotropin (hCG) injections combined with subcutaneous recombinant human follicle-stimulating hormone (FSH) injections or human menopausal gonadotropin (hMG), which contains equal amount of FSH and luteinizing hormone. In secondary hypogonadism with prepubertal onset, FSH needs to be included to induce testicular growth and spermatogenesis.

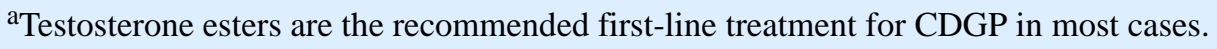


${ }^{b}$ Induction of fertility following treatment with GnRH may be less successful in men who have lower baseline testicular volumes, have previously received testosterone treatment and have not previously received treatment with GnRH or gonadotropins ${ }^{29,46-48}$. Because of these findings, recent studies have explored use of alternative methods to induce puberty with the ultimate question being whether these approaches increase subsequent fertility $^{49,50}$. Box based on data originally presented in REF. ${ }^{105}$. 


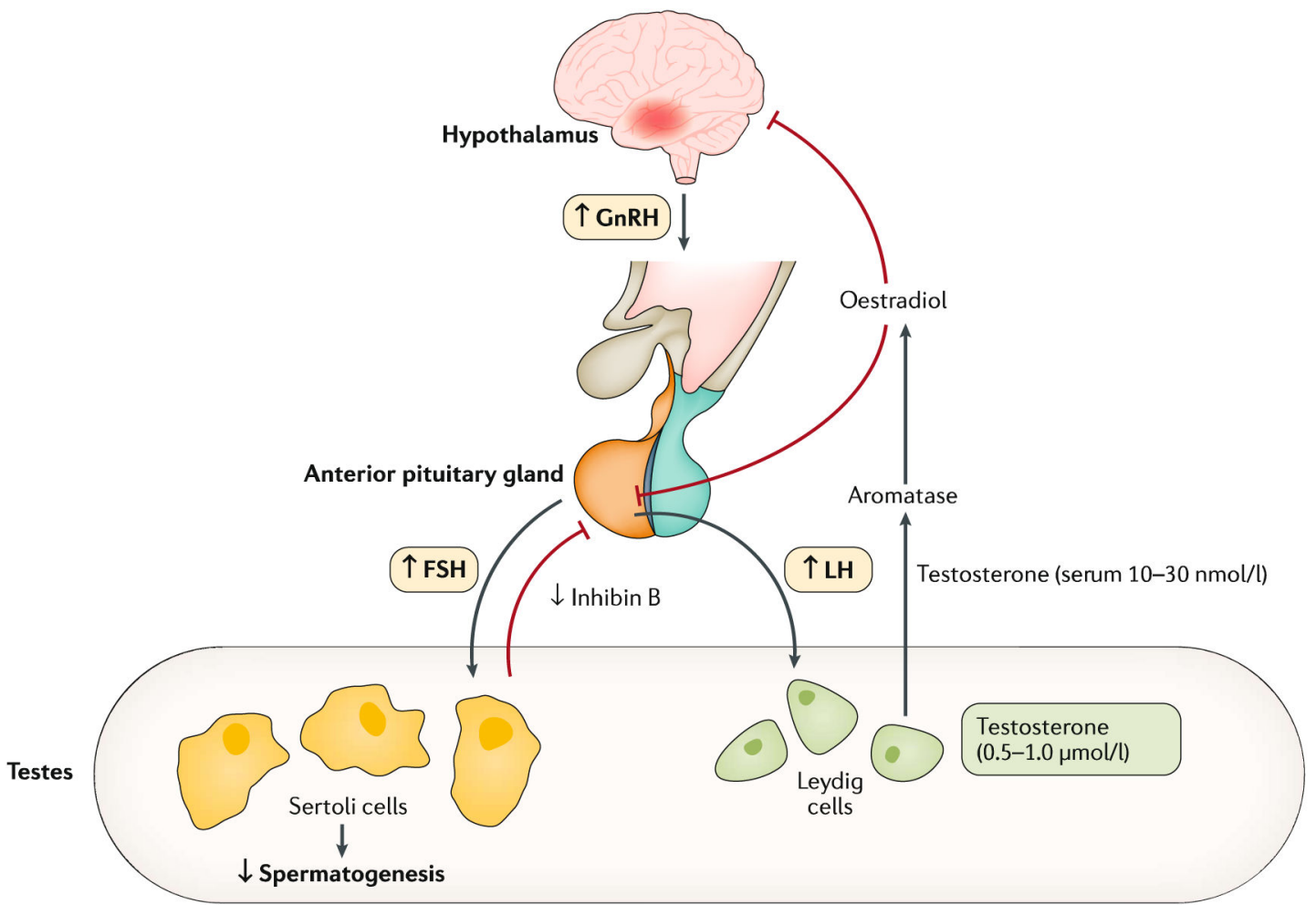

Fig. 1 |. The hypothalamic-pituitary-gonadal axis.

Both testosterone synthesis and male fertility result from the delicate coordination throughout the hypothalamic-pituitary-gonadal axis, thereby ensuring normal testicular function ${ }^{1}$. Gonadotropin-releasing hormone $(\mathrm{GnRH})$ stimulates the release of luteinizing hormone (LH) from the pituitary gland. This triggers the Leydig cells within the testes to respond by producing adequate levels of testosterone, which, in turn, exerts negative feedback control on the hypothalamus and pituitary gland. Likewise, GnRH stimulates the release of follicle-stimulating hormone (FSH) from the pituitary gland. This triggers and sustains the spermatogenesis within the exocrine part of the testes. The testes contribute $>95 \%$ of total circulating testosterone in the postpubertal male; testosterone is secreted into the circulation down a concentration gradient, where it equilibrates between protein-bound (98\%) and free hormone (1-2\%) fractions. Circulating testosterone and other sex hormones are bound either to low-affinity, high-availability proteins (primarily albumin) or to the highaffinity glycoprotein sex hormone-binding globulin (SHBG). These binding proteins play an important part in regulating the transport, distribution, metabolism and biological activity of the sex hormones ${ }^{231,232}$. Conditions that alter SHBG levels (for instance, ageing, obesity, insulin resistance and liver disease) influence free testosterone levels. The free hormone fraction is postulated to be the biologically active form of testosterone ${ }^{1,231,232}$. Testosterone secretion varies throughout the day and is usually the highest in the morning. Hence, samples to determine testosterone levels need to be taken in the morning. Figure adapted from REF. ${ }^{233}$, Springer Nature Limited. 


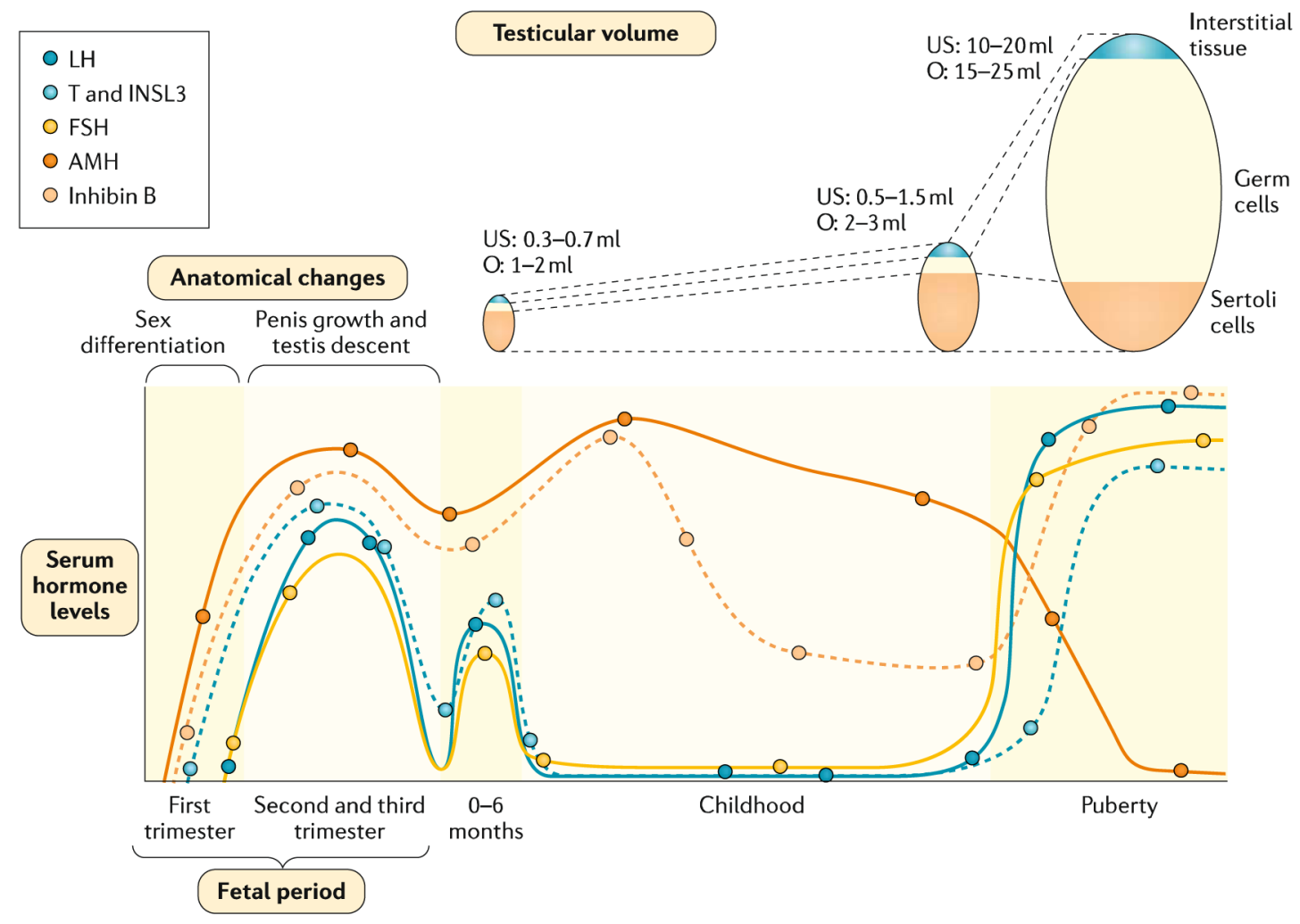

Fig. 2 |. Anatomical changes and serum hormone levels associated with male sex determination and maturation.

In the fetal period, testicular hormones begin to be secreted independently of fetal pituitary gonadotropins in the first trimester of fetal life and drive fetal differentiation of the genitalia. In the second and third trimesters, growth of the genitalia and testicular descent are stimulated by androgen secretion dependent on fetal luteinizing hormone (LH). In the postnatal period, testicular volume increases during childhood owing essentially to Sertoli proliferation. After the postnatal activation in the 0-6-month period (usually called 'minipuberty'), serum levels of gonadotropins and testosterone (T) decline, but those of the Sertoli cell markers anti-Mullerian hormone (AMH) and inhibin B persist at clearly detectable levels. During puberty, testicular volume increases dramatically owing to spermatogenic development, secondary to gonadotropin and T action. Sertoli cell markers show opposite profiles: AMH is inhibited by $\mathrm{T}$ whereas inhibin B is upregulated by folliclestimulating hormone (FSH) and germ cells. INSL3, insulin-like factor 3; O, testicular volume measured by comparison to Prader's orchidometer; US, testicular volume measured by ultrasonography. 


\section{a Primary hypogonadism b Secondary hypogonadism}

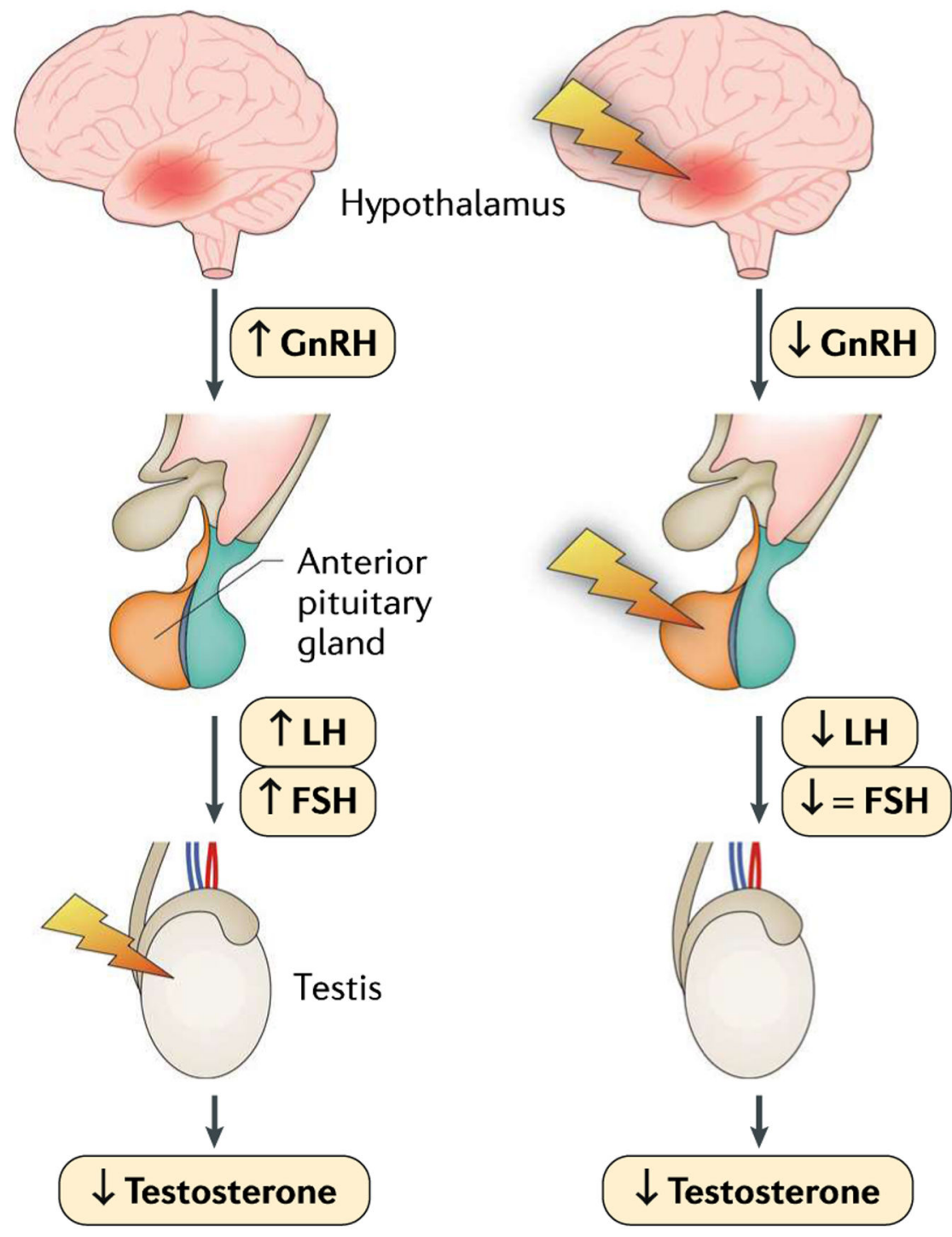

Fig. 3 |. Pathophysiology of hypogonadism.

Hypogonadism may be caused by a primary testicular pathology (primary hypogonadism, otherwise known as hypergonadotropic hypogonadism, which is defined as low testicular hormones, with high gonadotropins) resulting from malfunction at the level of the testes due to a genetic cause, injury, inflammation or infection (panel a). Conversely, central defects of the hypothalamus or the pituitary gland lead to secondary hypogonadism (also called central hypogonadism or hypogonadotropic hypogonadism, which is defined as low testicular hormones, with low or normal gonadotropins), which is most often caused by genetic defects, neoplasm or infiltrative disorders (panel b). FSH, follicle-stimulating hormone; GnRFI, gonadotropin-releasing hormone; LH, luteinizing hormone. Adapted with permission from REF. ${ }^{31}$, Oxford University Press. 


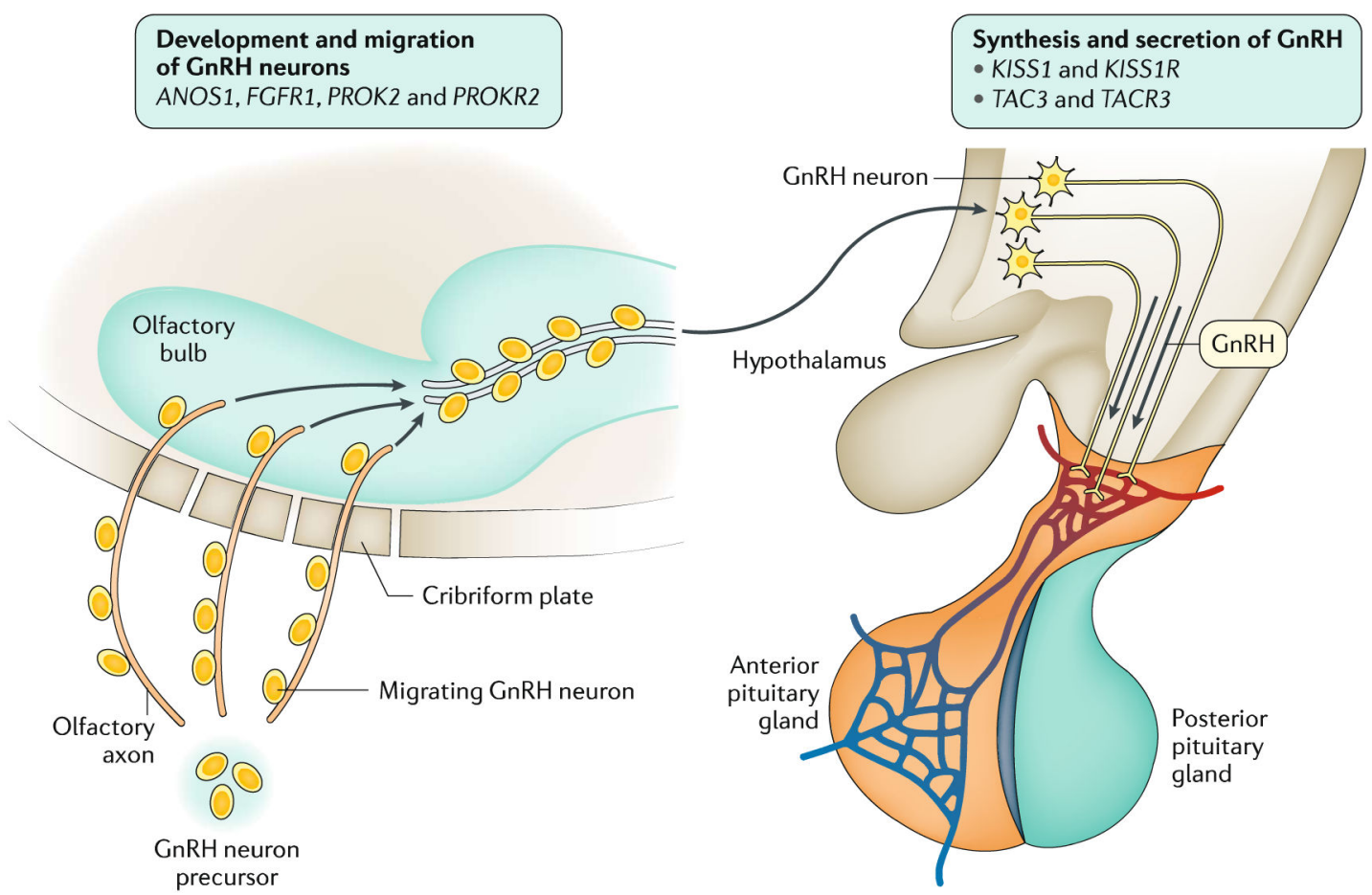

Fig. 4 |. Pathophysiology of congenital secondary hypogonadism.

Genes associated with congenital secondary hypogonadism. GnRH, gonadotropin-releasing hormone. 

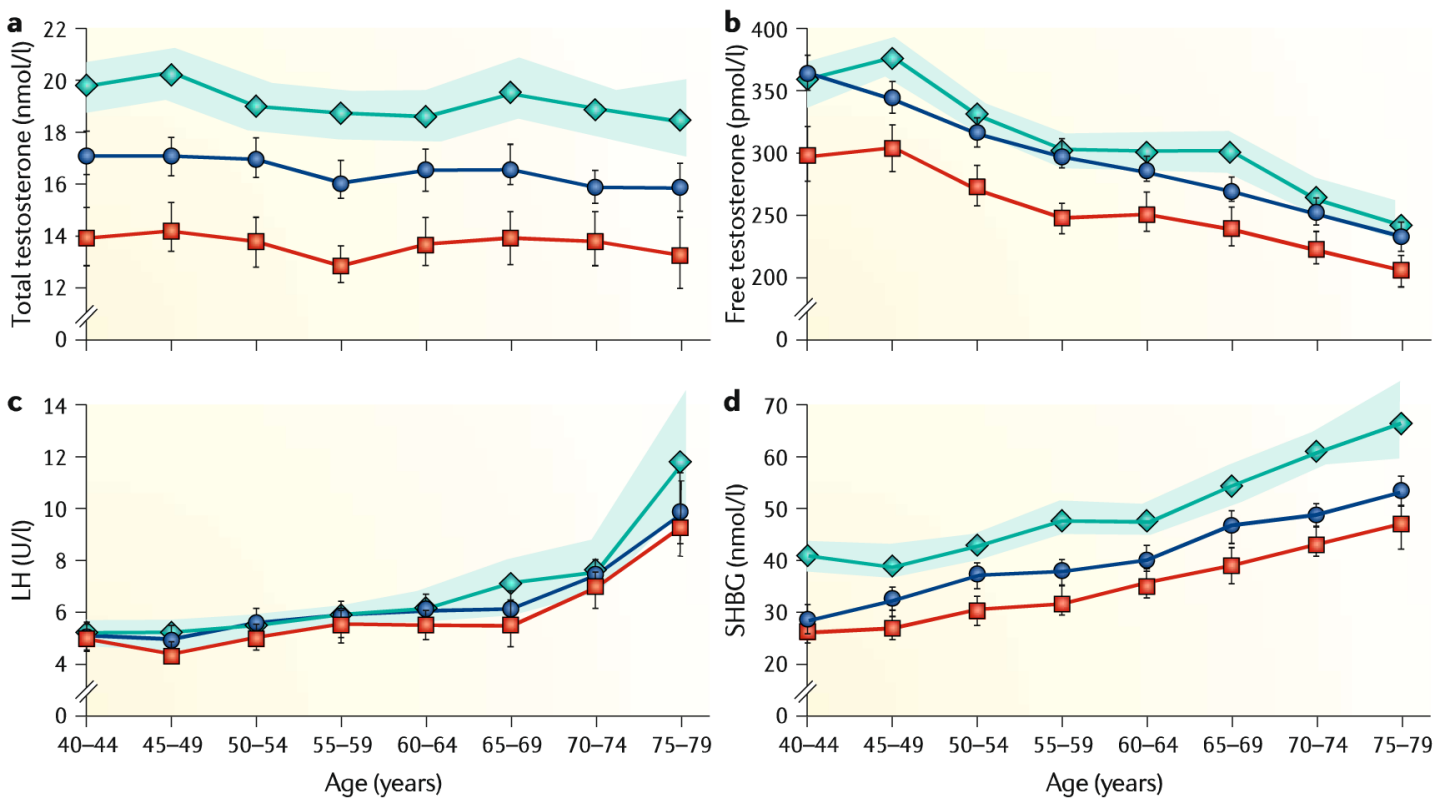

$\diamond \mathrm{BMI}<25 \mathrm{~kg} / \mathrm{m}^{2} \quad$ O BMI $25-29 \mathrm{~kg} / \mathrm{m}^{2} \quad \square \mathrm{BMI} \geq 30 \mathrm{~kg} / \mathrm{m}^{2}$

Fig. 5 |. Relationship between age, BMI and reproductive hormones.

The graphs present mean levels of total and calculated free testosterone, luteinizing hormone (LH) and sex hormone-binding globulin (SHBC). a | Total testosterone is reduced in overweight and obese men compared with nonobese men at all ages. b|Free testosterone, similar to total testosterone, is reduced in overweight and obese men compared with nonobese men at all ages. $\mathbf{c} \mid \mathrm{LH}$ increases with age but is not associated with body mass index (BMI). d | SHBC increases with age. For total testosterone and SHBC, no interaction between BMI and age were found, whereas free testosterone showed an interaction between BMI and age. The data were derived from a cohort of 3,220 men aged 40-79 years recruited in the European Male Ageing Study (EMAS) study. Shaded areas and vertical lines represent the $95 \%$ CI. Adapted with permission from REF. ${ }^{76}$, Oxford University Press. 


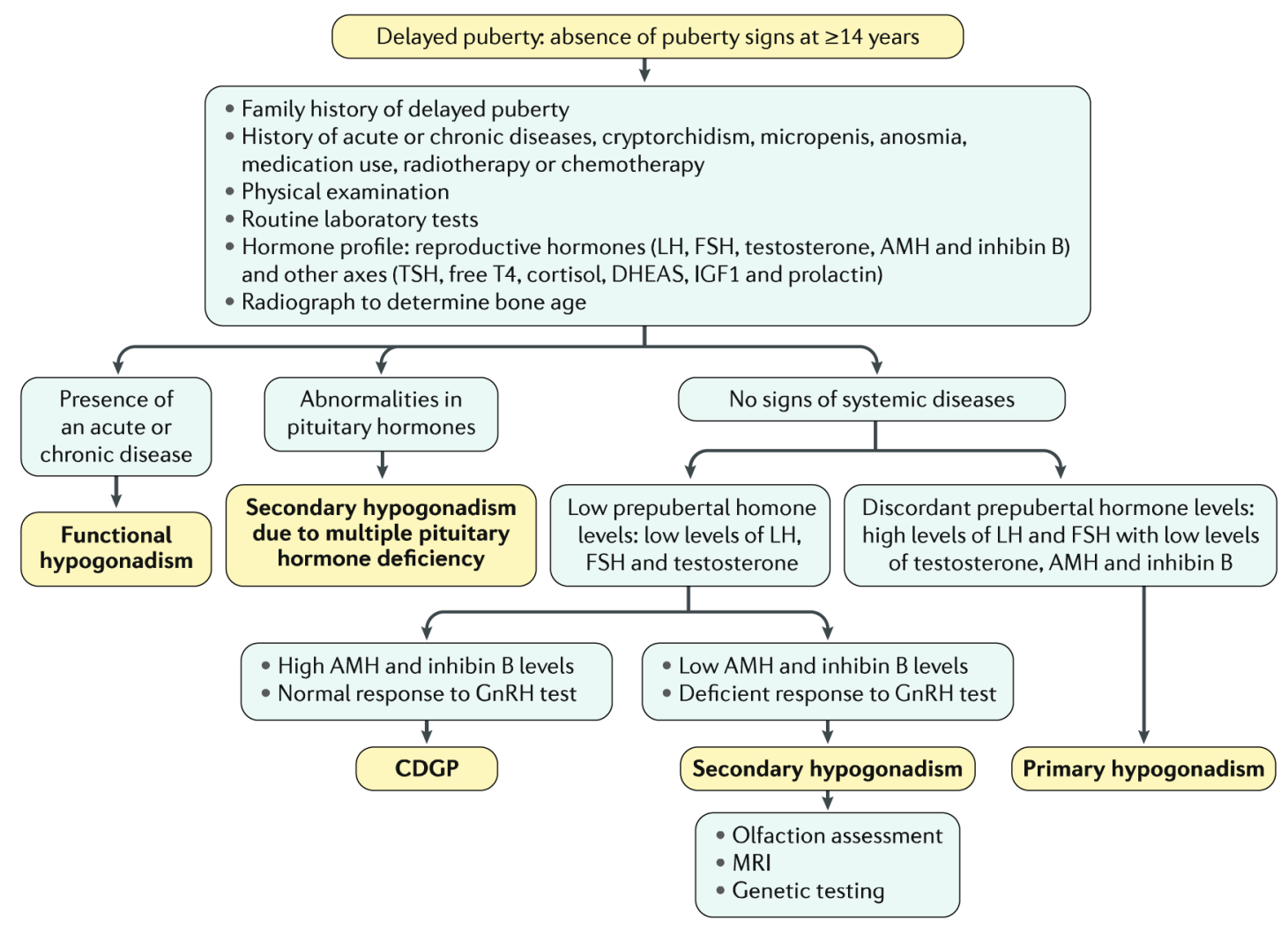

Fig. 6 |. Diagnostic algorithm for hypogonadism in pubertal age.

Diagnostic flowchart in a boy presenting with no signs of pubertal development by 14 years of age. Tests mentioned may help to distinguish among and/or confirm diagnoses, but the full battery is not recommended or warranted in all cases and may not lead to a conclusive diagnosis. The main text describes considerations regarding test use. AMH, anti-Müllerian hormone; CDGP, constitutional delay of growth and puberty; DHEAS, dehydroepiandrosterone; FSH, follicle-stimulating hormone; GnRH, gonadotropin-releasing hormone; IGF1, insulin-like growth factor 1; LH, luteinizing hormone; T4, thyroxine; TSH, thyroid-stimulating hormone. 


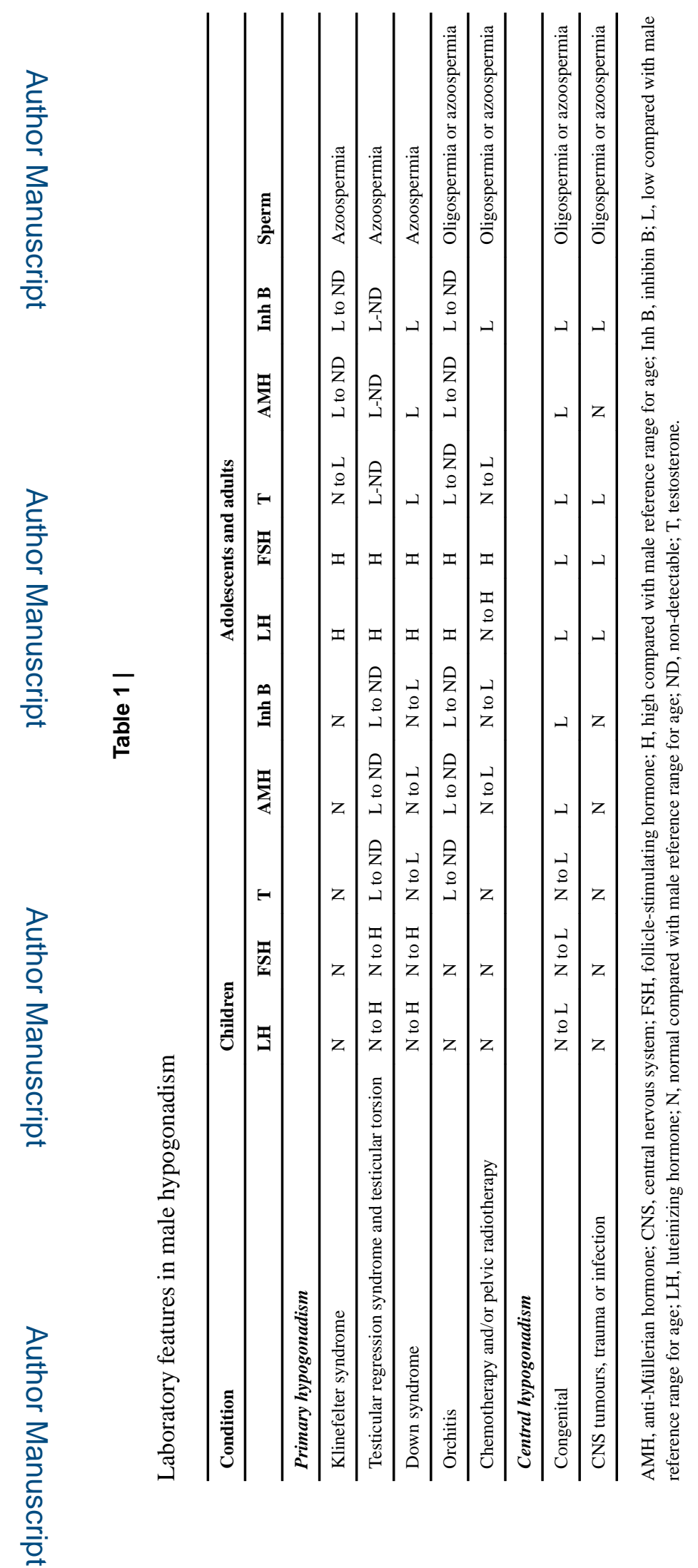

Nat Rev Dis Primers. Author manuscript; available in PMC 2020 January 06. 
Table 2 |

Laboratory cut-off values for biochemical late-onset hypogonadism

\begin{tabular}{|c|c|c|c|}
\hline Guideline & Total testosterone; nmol/l (ng/ml) & $\begin{array}{l}\text { Calculated free; testosterone } \\
\text { pmol/l }(\mathbf{p g} / \mathrm{ml})\end{array}$ & Ref. \\
\hline American Urological Association & $10.4(3.0)$ & NA & 234 \\
\hline British Society for Sexual Medicine & $\begin{array}{l}\text { - Mild: } 12.1(3.5) \\
\text { - Severe: } 8.0(2.31)\end{array}$ & $\begin{array}{l}\cdot \text { NA } \\
\cdot 225(65)\end{array}$ & 113 \\
\hline Canadian Endocrine Society & $\begin{array}{l}\text { Depending on reference values in local } \\
\text { laboratory }\end{array}$ & NA & 235 \\
\hline European Association of Urology & $12.1(3.5)$ & $243(70)$ & 120 \\
\hline Endocrine Society & $9.2(2.64)$ & NA & 112 \\
\hline International Society for Sexual Medicine & $12.0(3.5)$ & NA & 45 \\
\hline $\begin{array}{l}\text { International Society for the Study of the Aging } \\
\text { Male }\end{array}$ & $12.1(3.5)$ & $243(70)$ & 236 \\
\hline
\end{tabular}

NA, not applicable. 